\title{
Riqueza de especies y tipos funcionales: su relación en bosques de oyamel del Monte Tláloc, Estado de México
}

\section{Species richness and functional types: their relation in sacred-fir forests of Mount Tlaloc, Mexico State}

\author{
Cristóbal Sánchez-Sánchez!', Fernando Paz-Pellat²*, Patricia Hernández-de la Rosa', Alma Velázquez-Rodríguez, \\ Heike Vibrans`, Jesús Vargas-Hernández', Juan Ignacio Valdez-Hernández' y J. René Valdez-Lazaldel
}

Colegio de Postgraduados. Postgrado en Ciencias Forestales. Campus Montecillo. Montecillo, Estado de México, México.

2 GRENASER, Colegio de Postgraduados. Campus Montecillo. Montecillo, Estado de México, México.

\author{
Universidad Autónoma del Estado de México. \\ Campus El Cerrillo. Facultad de Ciencias. Estado de \\ México. \\ ${ }^{4}$ Colegio de Postgraduados. Campus Monteci- \\ Ilo. Postgrado en Botánica. Montecillo, Estado de \\ México, México.
}

\section{RESUMEN}

La diversidad de plantas vasculares está relacionada con la productividad y resiliencia de los bosques, por lo tanto, el desarrollo de indicadores para su caracterización es fundamental. La riqueza de especies vasculares $(S)$ ha mostrado ser un buen indicador de la diversidad de otros grupos biológicos. Asimismo, se ha propuesto que la riqueza de tipos funcionales de plantas $\left(\mathrm{S}_{\mathrm{g}}\right)$, se correlaciona significativamente con la riqueza de especies y, por lo tanto, puede ser usada como un indicador sustituto. En este trabajo se analiza la relación riqueza-área de muestreo $(\mathrm{A})$, con bases teóricas y datos empíricos para proponer un marco teórico generalizado de las relaciones entre los parámetros de las curvas: S-A, $\mathrm{g}_{\mathrm{g}}-\mathrm{A}$ y S-S. Se evalúa el modelo potencial y el logarítmico de las curvas de acumulación riqueza-área de especies de plantas vasculares y tipos funcionales, estos últimos caracterizados mediante una clasificación basada en 36 atributos funcionales en 15 sitios de muestreo de $1000 \mathrm{~m}^{2}$ en bosques de oyamel del Monte Tláloc, Estado de México. Los resultados muestran estimaciones de riqueza equiparables con las mediciones directas usando solamente un tipo de riqueza para estimar el otro. Esto puede simplificar considerablemente la obtención de indicadores de diversidad en inventarios de plantas vasculares.

Palabras Clave: Abies religiosa, espacios paramétricos, estimaciones simplificadas, indicadores de diversidad, relación de exponentes.

\section{ABSTRACT}

The diversity of vascular plants is related to the productivity and resilience of forests, and therefore, the development of indicators for its characterization is fundamental. The richness of vascular species (S) has been shown to be a good indicator of other biological groups diversity. It has also been proposed that the type of functional plant richness $\left(\mathrm{S}_{\mathrm{g}}\right)$ significantly correlates with the species richness and, therefore, can be used as a substitute indicator. This paper analyzes the relationship between richness and sampling area (A), with theoretical and empirical data, to propose a generalized theoretical framework of the relations between the parameters of the curves: $\mathrm{S}$-A, $\mathrm{S}_{\mathrm{g}}-\mathrm{A}$ and S-S. The power and logarithmic models of functional vascular plant types and richness-area accumulation curves are assessed, characterizing functional types through a classification based on 36 functional attributes, in 15 sampling sites of $1000 \mathrm{~m}^{2}$ in the sacred-fir forests of Mount Tlaloc, State of Mexico. Results show richness estimates comparable with the direct measurements, obtained by using only one type of richness to estimate the other. This can greatly simplify the implementation of diversity indicators in vascular plant surveys.

KEYwORDs: Abies religiosa, parameter spaces, simplified estimates, diversity indicators, relations among exponents. 


\section{Introducción}

La conservación e incremento de la biodiversidad es uno de los objetivos de la Convención sobre la Diversidad Biológica (CBD, por sus siglas en inglés), dentro del marco de la Organización de las Naciones Unidas (United Nations, Treaty Series [UNTC] 1992; Cooper y Noonan-Mooney, 2013). La CBD propone el uso de indicadores para evaluar la biodiversidad y revisar el cumplimiento de sus metas (Convention on Biological Diversity [CBD] 2010). El concepto de biodiversidad es muy amplio para su implementación práctica en la gestión de recursos naturales y marcos regulatorios (Noss, 1990), por ello es necesario el desarrollo de indicadores costo-efectivos, coherentes con lo que se busca evaluar, ya que su selección tiene consecuencias preponderantes en los resultados (Dobson, 2005; Mace y Baillie, 2007).

La diversidad vegetal de los bosques, representada por el número de especies (riqueza), está relacionada con la productividad forestal y su valor para la conservación (Thompson, Mackey, McNulty y Mosseler, 2009; Liang et al., 2016), por esta razón es importante caracterizarla adecuadamente. El uso de grupos de especies como indicadores para evaluar en forma indirecta la diversidad de otros grupos biológicos es común (Lewandowski, Noss y Parsons 2010; Lindenmayer y Likens, 2011) y debe basarse en el conocimiento de sus relaciones ecológicas, sin embargo, generalmente las aproximaciones obtenidas son insuficientes (Saetersdal y Gjerde, 2011; Gao, Nielsen y Hedblom, 2015). Se considera que las evaluaciones indirectas de diversidad a través de riqueza de especies con una efectividad mayor a $50 \%$ son aceptables (Lewandowski et al., 2010).

El extenso uso de la riqueza de plantas vasculares (S) como indicador de la diversidad general de un área determinada se debe, entre otras cosas, a que son relativamente fáciles de medir, constituyen la mayor parte de la biomasa forestal, generan hábitat para otros organismos y se relacionan ecológicamente con diversos grupos biológicos (Santi et al., 2010; Lindenmayer y Likens, 2011; Gao et al., 2015). Adicionalmente, las especies vasculares tienen una extensa variedad de mecanismos de respuesta al ambiente, de modo que es posible analizar su distribución en términos de factores ambientales a partir de la medición de sus atributos morfológicos y fisiológicos (Dumortier et al., 2002; Ohlemüller et al., 2004).

La riqueza de plantas vasculares (en términos de presencia-ausencia, no de abundancia), es uno de los indicadores más usados en las evaluaciones de diversidad de bosques y otros tipos de vegetación (National Research Council [NRC] 2000; Newton y Kapos, 2002). La distribución de estas especies (totales y nativas), bajo diferentes usos del suelo, se utiliza para calcular índices y analizar el efecto del cambio de uso de suelo, usualmente tomando como referencia la vegetación sucesionalmente madura (NRC, 2000; Croezen, Bergsma, Clemens, Sevenster y Tulleners, 2011).

Specht (1972) teorizó condiciones de equilibro (etapa sucesional madura) de la vegetación en función de un equilibrio dinámico entre la demanda evaporativa de la atmósfera y la disponibilidad de agua del suelo. En la vegetación en equilibrio, la región climática (temperatura media y número de meses de crecimiento de la vegetación) y las condiciones de equilibrio dinámico son representadas por un coeficiente evaporativo k, y la riqueza de especies está relacionada en forma exponencial con dicho coeficiente (Specht y Specht, 1989 y 1993).

Dado que k se relaciona linealmente con la cobertura proyectiva foliar (Specht, 1972), la riqueza también se correlaciona con otros componentes del ecosistema. Por ejemplo, la riqueza de plantas vasculares ( $\mathrm{S}$ ) en sitios de vegetación en equilibrio, ha mostrado correlaciones con la riqueza de anfibios, serpientes, lagartijas, aves y pequeños mamíferos (Specht, 1994, 2012, Specht y Specht, 2013), por lo tanto, el número de especies vasculares puede considerarse como un buen indicador de la diversidad en general. Sin embargo, se ha argumentado que la riqueza de especies por sí misma no influye directamente en respuestas dinámicas de la vegetación a cambios ambientales, como lo hacen los grupos o tipos funcionales (Phillips, Hall, Gentry, Sawyer y Vásquez, 1994; Tilman, Knops, Wedin y Reich, 2002), entendidos como conjuntos de organismos que comparten ciertas características morfoló- 
gicas, fisiológicas y adaptativas, de respuesta al ambiente, independientemente de su afinidad taxonómica (Díaz y Cabido, 2001).

La clasificación de tipos funcionales depende del objetivo, escala y proceso ecosistémico o factor ambiental de interés (Gitay y Noble, 1977; Lavorel, McIntyre, Landsberg y Forbes, 1997), pero en general, los tipos funcionales de plantas son aquellos que responden de manera similar al ambiente o tienen efectos similares en el funcionamiento del ecosistema (Smith et al., 1993; Shugart, 1996; Gitay y Noble, 1997; Díaz y Cabido, 2001).

Un método de clasificación de tipos funcionales adecuado para inventarios es el propuesto por Gillison (Gillison, 1981; Gillison y Carpenter, 1997; Gillison, 2002; Gillison, 2006), ya que caracteriza a las especies de manera sencilla y práctica, en función de atributos de la estructura fotosintética (tamaño e inclinación de las hojas, clorotipo y tipo de hoja) y de la estructura vascular de soporte (forma de vida y tipo de raíz). La riqueza de tipos funcionales $\left(\mathrm{S}_{\mathrm{g}}\right)$ evalúa la redundancia funcional de las plantas vasculares en la vegetación. Gillison et al. (2003; 2013) mostraron que la relación $\mathrm{S} / \mathrm{S}_{\mathrm{g}}$ se asocia con la riqueza de especies de termitas, aves y escarabajos (Gillison y Liswanti, 2004), así como con el carbono de la biomasa aérea (Gillison et al., 2003; Gillison y Liswanti, 2004), aun cuando esta última relación muestra una saturación de $\mathrm{S} / \mathrm{S}_{\mathrm{g}}$ (después de un umbral).

La diversidad funcional tiene distintas métricas, de manera que no todas sus evaluaciones se correlacionan con la riqueza de especies (Díaz y Cabido, 2001; Hooper et al., 2005), en cuanto a la riqueza de tipos funcionales de plantas, se ha documentado una relación casi lineal con la riqueza de especies e independiente del área de muestreo que podría permitir estimar S cuando se conoce Sg (Gillison, 2016). La evaluación de la diversidad forestal requiere del uso de indicadores que caractericen los atributos primarios de composición, estructura y función de las plantas (Franklin, 1988; Noss, 1990). En este sentido, la riqueza de especies (S) evalúa la composición, la riqueza de tipos funcionales $\left(\mathrm{S}_{\mathrm{g}}\right)$ es una métrica de la función, y la razón
$\mathrm{S} / \mathrm{S}_{\mathrm{g}}$ evalúa, indirectamente, la estructura de los bosques (Gillison, 2016).

Los indicadores de diversidad son dependientes del área del muestreo (Rosenzweig, 1995), por lo tanto, es fundamental caracterizar las relaciones riqueza-área (S-A). Asimismo, la riqueza de tipos funcionales varía en función de la superficie muestreada (Gillison, 2002). Es por esto que la relación riqueza-área ha sido considerada como una de las pocas reglas generales en ecología (Schoener, 1976; Lawton, 1999; Lomolino, 2000) y ha sido aplicada en la evaluación del efecto de la pérdida de área (hábitat) por cambios de uso del suelo, así como en estimaciones de riqueza y extinción de especies (Tilman y Lehman, 1997; Tilman, May, Lehman y Nowak, 1994; Brooks et al., 2001; Pereira y Daily, 2006; Koh y Ghazoul, 2010; Rybicki y Hansi, 2013), particularmente en relación con el diseño de reservas ecológicas o áreas naturales protegidas (Tjørve, 2010).

\section{OBJETIVOS}

Esta investigación, desarrollada en bosques de oyamel en el Estado de México, tiene como objetivo evaluar el uso de la riqueza de tipos funcionales de plantas vasculares como un indicador de la riqueza de especies en diferentes escalas espaciales, mediante un marco teórico basado en matemáticas sólidas. Con base en los antecedentes teóricos presentados, se plantea que la relación de los parámetros de las correlaciones entre tipos funcionales, especies y áreas, permitirá cálculos de riqueza comparables con los ejercicios directos de medición, que permitirá simplificar el problema de estimaciones a escala.

\section{MATERIALES Y MÉTODOS}

\section{Fundamento teórico}

\section{Modelos de la relación riqueza de especies-área (S-A) y} sus ajustes estadísticos

El número de especies vasculares varía en función del área (A) de muestreo (Rosenzweig, 1995) (Fig. 1), y la relación 
riqueza-área (S-A) puede obtenerse mediante diferentes esquemas de muestreo, como los anidados, contiguos, los no contiguos, islas, entre otros (Scheiner, 2003), por lo tanto, de acuerdo con el método de muestreo se pueden obtener patrones que son producto del método o no tienen bases sólidas (Drakare, Lennon y Hillebrand, 2006; Palmer, McGlinn y Fridley, 2008; Dengler, 2008; Smith, 2010). De acuerdo con estas consideraciones, los siguientes análisis se basan en modelos de la relación S-A para el caso de muestreos anidados.

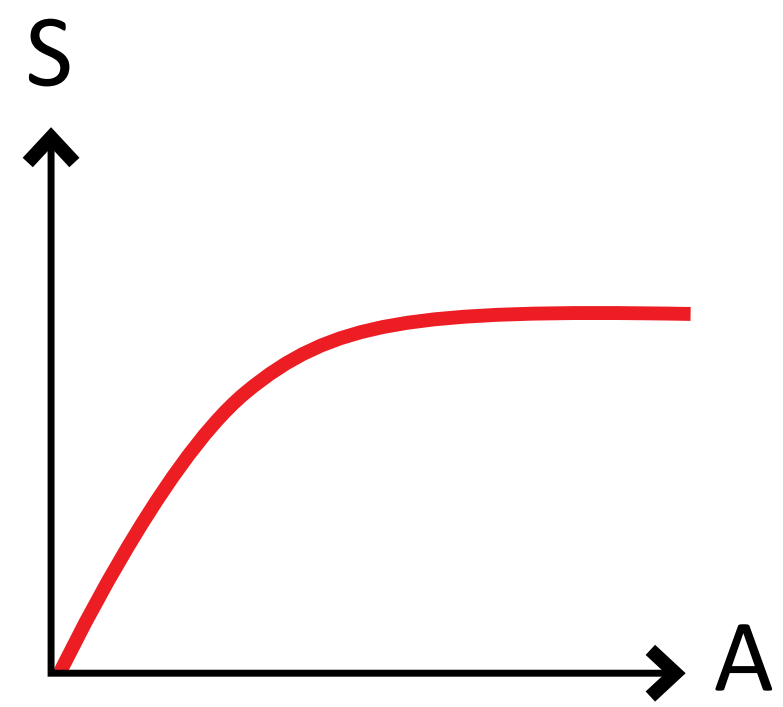

Figura 1. Relación riqueza de especies (S)-área (A).

Existen muchas propuestas matemáticas para explicar la relación S-A (Tjørve, 2003; Scheiner, 2003; Dengler, 2009; Tjørve, 2009), las cuales poden clasificarse en dos grupos: sin asíntota y con asíntota (Lomolino, 2000 y 2001). Los modelos asintóticos (sigmoides) han sido severamente criticados (Williamson, Gaston y Lonsdale, 2001 y 2002) por tener un soporte matemático débil (Triantis, Guilhaumon y Whittaker, 2012), por esta razón se ha recomendado el uso de modelos convexos como alternativa (p. ej. el potencial y el logarítmico).

Se han analizado los modelos que mejor se ajustan a datos experimentales (Connor y McCoy, 1979; Dengler, 2009, Triantis et al., 2012), y los más adecuados han sido el potencial (Arrehenius, 1921; Preston, 1962) y el logarítmico (Gleason, 1922; Hopkins, 1955):

$$
\begin{aligned}
& S=c A^{z} \\
& S=c+z \ln (A)
\end{aligned}
$$

El modelo potencial, ecuación (1), tiene intersección en $(0,0)$, a diferencia del modelo logarítmico con intersección en un valor negativo $(c<0$, generalmente), definiendo un área crítica $(\mathrm{A}>0)$ para la existencia de una sola especie (Lomolino, 2001). El modelo logarítmico se usó comúnmente en el pasado (p. ej. Specht y Specht, 1989), pero el modelo potencial se popularizó bajo la hipótesis de equilibrio (Preston, 1962; MacArthur y Wilson, 1967) de la teoría de biogeografía de islas, que sugiere que la biota de cualquier isla es un equilibrio dinámico entre la inmigración de nuevas especies y las tasas de extinción.

Se han evaluado diferentes explicaciones acerca del uso de los modelos S-A, principalmente el potencial, por diferentes autores (Connor y McCoy, 1979; McGuinness, 1984; Turner y Tjørve, 2005) sin mostrar explicaciones satisfactorias para las hipótesis específicas, de manera que se requieren argumentos multifactoriales para explicar los patrones encontrados y para justificar la omisión de otros modelos (Dengler, 2008; Smith, 2010).

El modelo potencial generalmente se ajusta mediante el uso de una transformación logarítmica:

$$
\ln (S)=\ln (c)+z \ln (A)
$$

El coeficiente de determinación $\left(\mathrm{R}^{2}\right)$ de la regresión lineal del modelo potencial de la ecuación (3), es diferente del que se obtiene al ajustar directamente el modelo de la ecuación (1) ajustado por regresión no lineal (Loehle, 1990; Cresswell, Vidal-Martinez y Crichton, 1995; Dengler, 2009); es por esto que no debe usarse para seleccionar un modelo de la relación S-A. El uso de ajustes por 
regresión lineal ordinaria para la ecuación (3) genera estimaciones sesgadas en relación a la media (Baskerville, 1972; Wright, 1981; Sprugel, 1983; McGuinness, 1984).

Para corregir el sesgo de la transformación logarítmica, bajo el supuesto de varianza constante de la variable transformada $S^{\prime}=\ln (S)$, el modelo puede ponerse como:

$$
\ln (S)=S^{\prime}=\ln (\mathrm{c})+z \ln (A)+\varepsilon
$$

donde el error $\varepsilon$ se distribuye normalmente:

$$
\varepsilon \sim \mathrm{N}\left(0, \sigma^{2}\right)
$$

con media cero y varianza del error (error estandar residual o EER) se define por:

$$
\sigma^{2}=\frac{1}{n-p} \sum_{i=1}^{n}\left(S_{i}^{\prime}-\hat{S}_{i}^{\prime}\right)^{2}
$$

donde

$\hat{S}_{i}^{\prime}$ es el valor estimado

$S_{i}^{\prime}$ el valor medido

$n$ el número de datos

p el número de parámetros del modelo $(\mathrm{p}=2)$

Las estimaciones de $S$, corregidas por el sesgo, están dadas por (Baskerville, 1972):

$$
\hat{S}_{i}=c A_{i}^{Z} \exp \left(\frac{\sigma^{2}}{2}\right)
$$

El término exponencial de la ecuación (7) es el factor de corrección (FC) que debe usarse para corregir el sesgo de usar la transformación logarítmica de la ecuación (1) para los ajustes por regresión lineal.

Adicionalmente al uso del coeficiente de determinación en el espacio aritmético (no el logarítmico), el ajuste estadístico de los modelos puede hacerse mediante la raíz del error cuadrático medio (RECM):

$$
R E C M=\sqrt{\frac{1}{n} \sum_{i=1}^{n}\left(\hat{S}_{i}-S_{i}\right)^{2}}
$$

\section{Patrones de la relación de los parámetros del modelo potencial y logarítmico}

La interpretación de los parámetros $c$ y $z$ del modelo potencial ha sido materia de discusión en la literatura (Gould, 1979; Connor y McCoy, 1979). El parámetro $z$, estable a escala (cambio de las unidades de medición), se ha discutido como función del coeficiente de determinación y la razón de las desviaciones estándar de $S$ y A, demostrando que para un coeficiente de determinación fijo, $z$ depende de la razón de las desviaciones estándar (Connor y McCoy, 1979). Otras interpretaciones de $z$ son dependientes de las hipótesis usadas, donde la de equilibrio plantea un valor “canónico" (Preston, 1962), que generalmente no es constante en los ajustes aplicados para plantas (Sala et al., 2005). El parámetro c generalmente se interpreta como parte del ajuste estadístico, dependiente de las unidades de medición usadas (Connor y McCoy, 1979; Gould, 1979).

Paz-Pellat, Odi-Lara, Cano-González, Bolaños-González y Zarco-Hidalgo (2009) demostraron que si las relaciones S-A se interceptan en un punto común, los parámetros de las rectas están relacionadas linealmente en el espacio loglog del modelo potencial (transformado a lineal) o el logaritmético del modelo logarítmico (transformado a lineal), entre sitios de distintas poblaciones de un mismo tipo de vegetación (Fig. 2). 


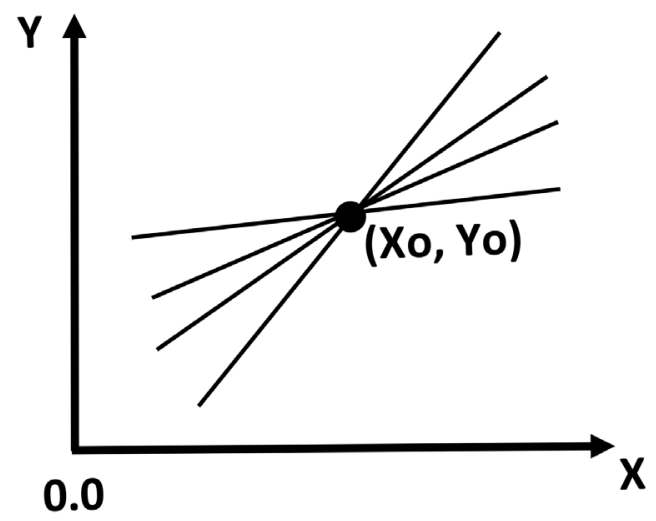

(a)

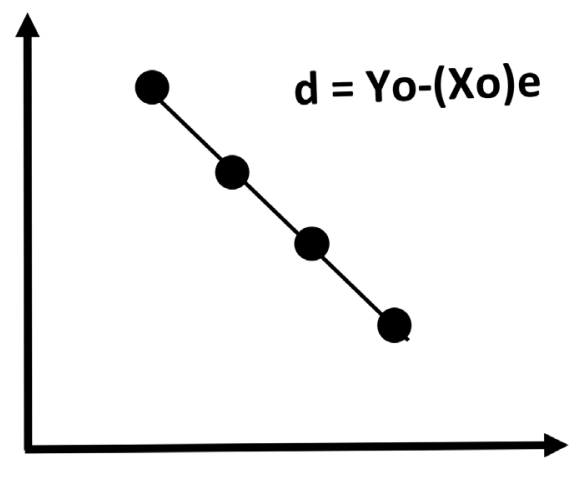

(b)

Figura 2. Relación entre los parámetros d y e, (a) líneas rectas que se interceptan en el punto (Xo, Yo) y (b) relación lineal entre d y e.

Las variables Y y X representan cualquier tipo de transformación que haga lineales los modelos.

En el modelo potencial, los parámetros se relacionan por (Lumer, 1936):

$$
\ln (c)=\ln (S o)-\ln (A o) z
$$

donde $\ln (A o)$ y $\ln (S o)$ representan el punto de intercepción de las líneas rectas en el espacio transformado logarítmicamente.

Para el modelo logarítmico, los parámetros están relacionados por:

$$
c=\text { So }-\ln (A o) z
$$

donde $\ln (A o)$ y $S o$ representan el punto de intersección de las líneas rectas en el espacio $\ln (A)-S$.

La relación inversa entre $d$ y $e(c \mathrm{y} z)$ (Fig. 2b) es para el caso de que la intersección de las líneas rectas cumpla $X o>0, \ln (X o)>\ln (1)$, en caso contrario la relación es directa (White y Gould, 1965).

La relación lineal entre los parámetros del modelo potencial y logarítmico (después de sus transformaciones a patrones lineales) (Fig. 2b), para el caso de que la inter- sección no sea en un punto común sino en una zona común, entonces la relación lineal tiene variaciones $\left(\mathrm{R}^{2}<1.0\right)$, dependientes del tamaño de la zona de intersección (Lumer, Anderson y Hersh, 1942).

La interpretación del parámetro $\mathrm{c}$ del modelo potencial $\left(c_{i}\right.$, $c$ inicial con la unidad de medida usada) puede efectuarse si se utiliza un factor de escala q para la variable A (área):

$$
S=c_{i}\left(\frac{A}{q}\right)^{z}=\left(\frac{c_{i}}{q^{z}}\right) A^{Z}
$$

Así, en un ajuste estadístico para estimar los parámetros $\mathrm{c}_{\mathrm{i}}$ y z usando unas unidades de medición dadas para el área de muestreo (p.ej. $\mathrm{m}^{2}$ ), si se transforman las unidades a (A/q) con $q>1$, entonces el valor de c varía en función del valor inicial $c_{i}, q$ y $z$, relación entre paréntesis de la ecuación (11) más a la derecha. Esta transformación puede usarse para mejorar la relación entre los parámetros $c$ y $z$ del conjunto de líneas rectas, haciendo que la intersección se aleje cada vez más del origen, $0 \mathrm{o} \ln (1)$.

El caso del modelo logarítmico se desarrolla de la siguiente manera: 


$$
S=c_{i}+z \ln \left(\frac{A}{q}\right)=\left[c_{i}-z \ln (q)\right]+z \ln (A)
$$

donde el parámetro $c$ depende del valor inicial $c_{i}, q$ y $z ; \mathrm{y}$ al igual que en el caso del modelo potencial, el valor de $\mathrm{q}$ puede usarse para mejorar la relación entre los paráme$\operatorname{tros} c$ y $z$.

\section{Relación entre S y Sg y sus patrones matemáticos}

Para establecer la relación entre $S$ y $S_{g}$ en el modelo potencial, es necesario definir las funciones riqueza-área para especies $(S-A)$ y tipos funcionales $\left(S_{g}-A\right)$ :

$$
\begin{aligned}
& S=c A^{z} \\
& S_{g}=c_{g} A^{z} g
\end{aligned}
$$

Al despejar A en ambas ecuaciones, e igualarlas, se obtiene:

$$
S=\left(\frac{c}{c_{g}^{z / z_{g}}}\right) S_{g}^{z / z_{g}}
$$

Para el caso de las relaciones $S$ - $A$ y $S_{g}-A$, en donde líneas rectas (espacio log-log) se intersectan en un punto o zona comun, se tiene:

$$
\begin{aligned}
& \ln (c)=\ln \left(A_{o}\right)-\ln \left(S_{0}\right) z \\
& \ln \left(c_{g}\right)=\ln \left(A_{0}\right)-\ln \left(S_{g 0}\right) z_{g}
\end{aligned}
$$

En el caso de la relación $S$ - $S_{g}$, se obtiene:

$$
\ln \left(\frac{c}{c_{g}^{z / z_{g}}}\right)=\ln \left(S_{0}\right)-\ln \left(S_{g 0}\right)\left(\frac{z}{z_{g}}\right)
$$

La ecuación (18) puede simplificarse para establecer las relaciones entre los parámetros:

$$
\mathrm{z}=\left[\frac{\ln \left(S_{0}\right)-\ln (c)}{\ln \left(s_{g_{0}}\right)-\ln \left(c_{g}\right)}\right] z_{g}
$$

$$
\ln (c)=\left[\ln \left(S_{0}\right)-\ln \left(S_{g 0}\right)\left(\frac{z}{z_{g}}\right)\right]+\left(\frac{z}{z_{g}}\right) \ln \left(c_{g}\right)
$$

$$
\ln (c)=\ln \left(S_{0}\right)+\left[\frac{\ln \left(c_{g}\right)-\ln \left(S_{g 0}\right)}{z_{g}}\right] z
$$

$$
\ln \left(c_{g}\right)=\ln \left(S_{g 0}\right)+\left[\frac{\ln (c)-\ln \left(S_{0}\right)}{z}\right] z_{g}
$$

Una alternativa para evitar despejar A en las ecuaciones (13) y (14) es plantear las relaciones riqueza-área como:

$$
\begin{aligned}
& A=a S^{b} \\
& A=a_{g} S_{g}^{b_{g}}
\end{aligned}
$$

Igualando las ecuaciones se obtiene:

$$
S=\left(\frac{a_{g}}{a}\right)^{1 / b} S_{g}^{b_{g / b}}
$$

La intersección de las líneas rectas de $A-S$ y $A-S_{g}$ en el espacio transformado está dada por:

$$
\begin{gathered}
\ln (a)=\ln \left(A_{0}\right)-\ln \left(S_{0}\right) b \\
\ln \left(a_{g}\right)=\ln \left(A_{0}\right)-\ln \left(S_{g 0}\right) b_{g}
\end{gathered}
$$

Para el caso de la relación $S$ - $S_{g}$, se obtiene:

$$
\ln \left(\frac{a_{g}}{a}\right)^{1 / b}=\ln \left(S_{0}\right)-\ln \left(S_{g 0}\right)\left(\frac{b_{g}}{b}\right)
$$

La ecuación (28) puede simplificarse para establecer las relaciones entre los parámetros:

$$
\mathrm{b}=\left[\frac{\ln \left(a_{g}\right)-\ln (a)}{\ln \left(\mathrm{S}_{0}\right)}\right]+\left[\frac{\ln \left(S_{g 0}\right)}{\ln \left(\mathrm{S}_{0}\right)}\right] b_{g}
$$




$$
\begin{aligned}
& \ln (a)=\left[\ln \left(S_{g 0}\right) b_{g}-\ln \left(S_{0}\right) b\right]+\ln \left(a_{g}\right) \\
& \ln (a)=\left[\ln \left(a_{g}\right)+b_{g} \ln \left(S_{g 0}\right)\right]-\ln \left(S_{0}\right) b \\
& \ln \left(a_{g}\right)=\left[\ln (a)+b \ln \left(S_{0}\right)\right]-\ln \left(S_{g 0}\right) b_{g}
\end{aligned}
$$

Ahora bien, en el caso del modelo logarítmico, los desarrollos son similares al caso del modelo potencial. Para el caso de la relación de riqueza-área se tiene:

$$
\begin{aligned}
& S=c+z \ln (A) \\
& S_{g}=c_{g}+z_{g} \ln (A)
\end{aligned}
$$

Despejando para $\ln (A)$ e igualando las ecuaciones, se obtiene:

$$
S=\left[c-c_{g}\left(\frac{z}{z_{g}}\right)\right]+\left(\frac{z}{z_{g}}\right) S_{g}
$$

que es una relación lineal entre $S$ y $S_{g}$.

\section{Relación entre exponentes}

En la sección anterior se establecieron las relaciones entre los parámetros ( $c$ y $z$ o $a$ y $b$ ) de las curvas riqueza-área, de manera que es necesario desarrollar un método para estimar las relaciones entre los exponentes $(z \circ b)$ de las curvas riqueza de especies-área, riqueza de tipos funcionales-área y entre ambos tipos de riqueza. En este sentido, es posible utilizar la relación entre exponenentes usando la teoría fractal desarrollada por Price, Enquist, y Savage (2007) y Zhang et al., (2016) en términos empíricos, considerando la evidencia experimental.

Si se considera un factor común t cualquiera que multiplique los exponentes de las relaciones área-riqueza, ecuaciones (23) y (24), es posible establecer las relaciones entre los exponentes como:

$$
t=\frac{1}{b_{g}-b}
$$

$$
\begin{aligned}
& A=a S^{t b}=a S^{\frac{b}{b^{-b}}}=a S^{\frac{1}{\left(b_{g} / b\right)-1}}=a S^{\frac{1}{(b / b g)}} \\
& A=a_{g} S_{g}^{t b_{g}}=a_{g} S_{g}^{\frac{b_{g}}{b_{g}-b}}=a_{g} S_{g}^{\frac{1}{1-\left(b / b_{g}\right)}}=a_{g} S_{g}^{\frac{1}{1-\frac{1}{\left(b_{g} / b\right)}}}
\end{aligned}
$$

Igualando las ecuaciones (37) y (38) se obtiene:

$$
S=\left(\frac{a_{g}}{a}\right)^{1 / t b} S_{g}^{b_{g / b}}=\left(\frac{a_{g}}{a}\right)^{\frac{b_{g}-b}{b}} S_{g}^{b_{g / b}}
$$

$S_{g}=\left(\frac{a}{a_{g}}\right)^{1 / t b_{g}} S^{b / b_{g}}=\left(\frac{a}{a_{g}}\right)^{\frac{b_{g}-b}{b_{g}}} S^{b / b_{g}}$

Las relaciones presentadas tienen exponentes comunes $(b /$ $b_{g}$ o $\left.b_{g} / b\right)$, por tanto, con la estimación de una relación $\left(A-S, A-S_{g}, S-S_{g}, S_{g}-S\right)$ es posible conocer los exponentes de todas las demás. Conocidos los exponentes, las constantes ( $\left.\begin{array}{lll}c & \mathrm{o} & a\end{array}\right)$ se estiman de las relaciones con los exponentes, para el caso de líneas rectas que se intersectan en un punto o zona común.

En términos de ajustes por regresión estadística, las ecuaciones (37) a (40) pueden ser expresadas como:

$$
\begin{aligned}
& A=m S^{p} \\
& A=m_{g} S_{g}^{p_{g}} \\
& S=a_{s g} S_{g}^{b_{s g}} \\
& S_{g}=a_{g s} S^{b_{g s}}
\end{aligned}
$$

donde las relaciones para el caso de intersección en un punto común de las últimas dos ecuaciones pueden escribirse como:

$$
\ln \left(a_{s g}\right)=\ln \left(S_{0}\right)-\ln \left(S_{g 0}\right) b_{s g}
$$




$$
\ln \left(a_{g s}\right)=\ln \left(S_{g 0}\right)-\ln \left(S_{0}\right) b_{g s}
$$

A partir de la ecuación (42) se estima $b_{s g}$ :

$$
b_{s g}=\frac{1}{1-\frac{1}{p_{g}}}
$$

y $a_{s g}$ de la ecuación (45) para obtener el valor de $S$ que le corresponde a una área $A$ de muestreo y un $S_{g}$, por tanto se puede calcular $S / S_{g}$.

En el caso de querer estimar $S g$, conocida la ecuación (41), entonces $b_{g s}$ se calcula de:

$$
b_{g s}=\frac{1}{1+\frac{1}{p}}
$$

y $a_{g s}$ de la ecuación (46) al aplicar la función exponencial.

\section{Área de estudio}

El área estudiada se encuentra en la vertiente occidental del Monte Tláloc, que forma parte de la región más septentrional de la Sierra Nevada, en el municipio de Texcoco, Estado de México. En esta zona se encuentran bosques de oyamel (Abies religiosa (Kunth) Schltdl. \& Cham.) en un intervalo altitudinal que va de los $3100 \mathrm{~m}$ a los $3500 \mathrm{~m}$, en suelos profundos, de textura media y ricos en materia orgánica. La precipitación anual es de $900 \mathrm{~mm}$ a $1000 \mathrm{~mm}$ y la temperatura media anual varía de $10^{\circ} \mathrm{C}$ a $12{ }^{\circ} \mathrm{C}$ (Sánchez-González y López-Mata, 2003).

Durante mayo y julio de 2017 se establecieron 15 sitios en los bosques de oyamel. La selección de sitios no fue aleatoria debido a las limitaciones impuestas por la accesibilidad. Se consideraron locaciones alejadas de los accesos principales del bosque y que no mostraran signos evidentes de disturbio por fuego, pastoreo o aprovechamiento forestal reciente. (Fig. 3, Tabla 1).
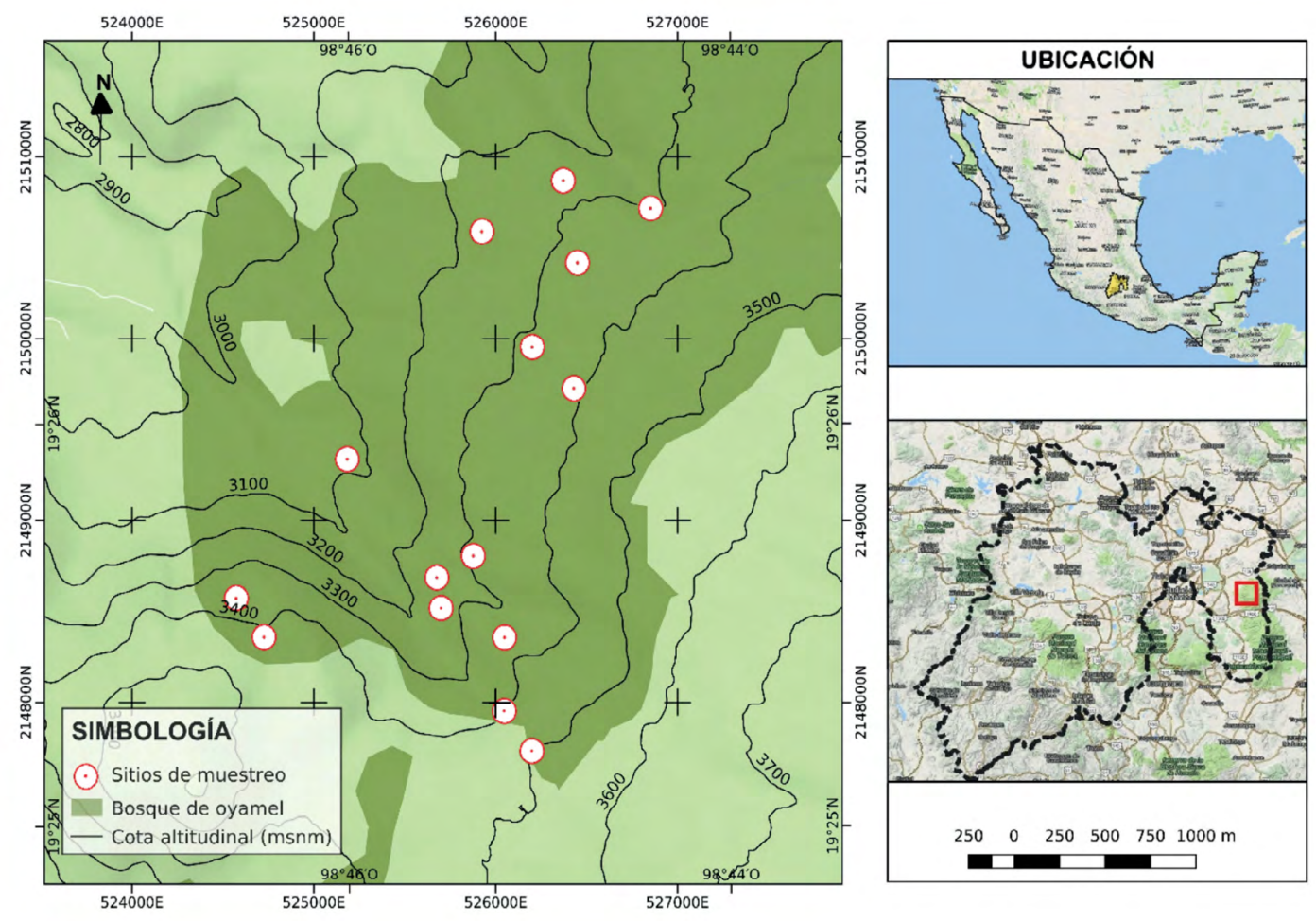

Figura 3. Ubicación de los sitios de muestreo en bosques de oyamel del Monte Tláloc, Estado de México. 
Tabla 1. Coordenadas geográficas de los sitios de muestreo en bosques de oyamel del Monte Tláloc, Estado de México.

\begin{tabular}{lccc}
\hline Sitio & Latitud & Longitud & Altitud $(m)$ \\
\hline 1 & $19^{\circ} 26^{\prime} 38.45^{\prime \prime}$ & $-98^{\circ} 45^{\prime} 1.34^{\prime \prime}$ & 3318 \\
2 & $19^{\circ} 26^{\prime} 18.33^{\prime \prime}$ & $-98^{\circ} 45^{\prime} 36.32^{\prime \prime}$ & 3082 \\
3 & $19^{\circ} 26^{\prime} 01.00^{\prime \prime}$ & $-98^{\circ} 45^{\prime} 12.59^{\prime \prime}$ & 3296 \\
4 & $19^{\circ}-25^{\prime} 51.60^{\prime \prime}$ & $-98^{\circ} 45^{\prime} 18.52^{\prime \prime}$ & 3233 \\
5 & $19^{\circ} 25^{\prime} 57.09^{\prime \prime}$ & $-98^{\circ} 45^{\prime} 19.46^{\prime \prime}$ & 3232 \\
6 & $19^{\circ} 25^{\prime} 33.18^{\prime \prime}$ & $-98^{\circ} 45^{\prime} 06.69^{\prime \prime}$ & 3391 \\
7 & $19^{\circ} 25^{\prime} 46.52^{\prime \prime}$ & $-98^{\circ} 45^{\prime} 52.07^{\prime \prime}$ & 3398 \\
8 & $19^{\circ} 25^{\prime} 53.30^{\prime \prime}$ & $-98^{\circ} 45^{\prime} 57.32^{\prime \prime}$ & 3336 \\
9 & $19^{\circ} 26^{\prime} 30.84^{\prime \prime}$ & $-98^{\circ} 44^{\prime} 53.56^{\prime \prime}$ & 3374 \\
10 & $19^{\circ} 26^{\prime} 53.39^{\prime \prime}$ & $-98^{\circ} 44^{\prime} 52.82^{\prime \prime}$ & 3332 \\
11 & $19^{\circ} 27^{\prime} 03.09^{\prime \prime}$ & $-98^{\circ} 44^{\prime} 39.12^{\prime \prime}$ & 3264 \\
12 & $19^{\circ} 27^{\prime} 08.09^{\prime \prime}$ & $-98^{\circ} 44^{\prime} 55.54^{\prime \prime}$ & 3257 \\
13 & $19^{\circ} 26^{\prime} 59.09^{\prime \prime}$ & $-98^{\circ} 45^{\prime} 10.86^{\prime \prime}$ & 3222 \\
14 & $19^{\circ} 25^{\prime} 26.08^{\prime \prime}$ & $-98^{\circ} 45^{\prime} 1.495^{\prime \prime}$ & 3481 \\
15 & $19^{\circ} 25^{\prime} 46.31^{\prime \prime}$ & $-98^{\circ} 45^{\prime} 6.625^{\prime \prime}$ & 3372 \\
\hline
\end{tabular}

\section{Técnicas de muestreo}

Los sitios de muestreo tuvieron una superficie circular de $1000 \mathrm{~m}^{2}$ con cinco subunidades concéntricas de $12.56 \mathrm{~m}^{2}$, $100 \mathrm{~m}^{2}, 200 \mathrm{~m}^{2}, 400 \mathrm{~m}^{2}$ y $1000 \mathrm{~m}^{2}$, de acuerdo con el diseño sugerido por Pearson, Brown y Birdsey (2007) y el manual de muestreo del Programa Mexicano del Carbono [PMC] (2015) para muestreos forestales y de carbono (Fig. 4).

Se registró la presencia de todas las especies de plantas vasculares en cada una de las cinco subunidades del sitio de muestreo, comenzando por el círculo interior de $12.56 \mathrm{~m}^{2}$ hasta llegar a la subunidad más externa (PMC, 2015). Se describió el tipo funcional de cada especie de acuerdo con el sistema de clasificación funcional desarrollado por Gillison (2002), el cual registra, la configuración espacial y temporal del tejido foliar y vascular de las plan- tas evaluadas, así como aptitudes relacionadas con su supervivencia y respuesta al ambiente.

El método de Gillison (2002, 2006) describe cada especie como un modelo funcional de dos componentes: su estructura fotosintética y su sistema vascular de soporte. La estructura fotosintética se caracteriza de acuerdo con la unidad foliar funcional más repetitiva, atendiendo a su tamaño, inclinación, clorotipo y forma. El sistema vascular de soporte es descrito con base en las formas de vida de Raunkiær (1934) y una clasificación de sistemas radicales adventicios. Los atributos funcionales evaluados constan de 36 caracteres, denominados elementos funcionales (Tabla 2), de manera que las especies y morfoespecies con la misma combinación de elementos funcionales conforman un tipo funcional (Gillison, 2002; Gillison, 2006). 


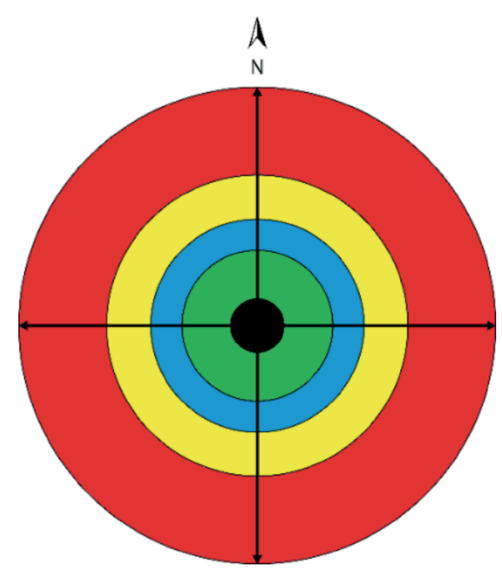

\begin{tabular}{|c|c|c|c|c|c|}
\hline Círculo & $\bullet$ & 0 & 0 & 0 & 0 \\
\hline $\begin{array}{l}\text { Radios de las } \\
\text { subunidades (m) }\end{array}$ & 2 & 5.65 & 7.98 & 11.28 & 17.85 \\
\hline $\begin{array}{l}\text { Área acumulada } \\
\left(\mathrm{m}^{2}\right)\end{array}$ & $\begin{array}{c}12.5 \\
6\end{array}$ & 100 & 200 & 400 & 1000 \\
\hline
\end{tabular}

Figura 4. Diseño del sitio de muestreo y medidas de sus subunidades.

Se fotografiaron y registraron las especies en floración o fructificación, mediante los métodos estándar (Lot y Chiang, 1986). La identificación taxonómica de gabinete se llevó a cabo con ayuda de literatura especializada. La nomenclatura de las especies se basó en el sistema de clasificación taxonómica de angiospermas (Byng et al., 2016). Para la nomenclatura de gimnospermas y helechos se consultó el proyecto de flora mundial Trópicos, del Missouri Botanical Garden [MBG] (2018).

\section{RESULTADOS}

Se identificaron 100 especies, pertenecientes a 72 géneros y 35 familias. Solo se documentó una especie del orden Polypodiales (Asplenium monanthes L.), y el oyamel fue la única gimnosperma registrada. Las angiospermas están representadas por 98 especies, 14 pertenecientes a la clase Liliopsida y 84 a la clase Magnoliopsida. Las familias mejor representadas fueron Asteraceae (24 spp.), Ericaceae (9 spp.), Poaceae (9 spp.), Orchidaceae y Rosaceae, ambas con 5 especies y Lamiaceae (4 spp.) (Fig. 5). La densidad promedio de árboles de más de $7.5 \mathrm{~cm}$ de diámetro normal por sitio fue de 32.9 árboles, predominantemente A. religiosa, con un valor promedio de $43 \mathrm{~cm}$ de diámetro normal.
Se registraron 55 tipos funcionales de plantas, los 15 más frecuentes están constituidos por 62 especies que representan $80 \%$ de los registros de frecuencias del muestreo. El número promedio de tipos funcionales por sitio de muestreo $\left(1000 \mathrm{~m}^{2}\right)$ fue de 19.8 , mientras que la riqueza de especies fue de 30.2 en promedio.

Las curvas de acumulación de especies y de tipos funcionales en relación con las áreas (A) de muestreo, se ajustaron mediante una regresión lineal ordinaria para cada sitio, con corrección de sesgos para el caso de la transformación logarítmica del modelo potencial. La tabla 3 muestra los resultados para el caso de riqueza de especies (S) y la tabla 4 para riqueza de tipos funcionales $\left(\mathrm{S}_{\mathrm{g}}\right)$.

El modelo potencial resultó ligeramente más significativo ( $\mathrm{R}^{2}$ y $\mathrm{RECM}$ ) al modelo logarítmico, aunque en casos particulares este último modelo presentó mejores ajustes.

En las tablas 3 y 4 se muestran los coeficientes de determinación del modelo potencial en el espacio log-log y el aritmético. Se puede observar que el aritmético fue el espacio correcto para decidir cuál modelo S-A es mejor. La ecuación (7) (Tabla 3 y 4), presentó valores bajos del factor de corrección (FC), de manera que no se intentó corregir las constantes estimadas del modelo potencial. 
Tabla 2. Atributos y elementos funcionales del sistema de clasificación de Gillison (2006) para plantas vasculares.

\begin{tabular}{|c|c|c|c|}
\hline $\begin{array}{l}\text { Componentes del modelo } \\
\text { funcional }\end{array}$ & Atributos funcionales & $\begin{array}{l}\text { Elementos } \\
\text { funcionales }\end{array}$ & Descripción \\
\hline \multirow{24}{*}{ Cubierta fotosintética } & \multirow{10}{*}{ Tamaño foliar } & $n r$ & Unidad foliar no repetida \\
\hline & & pi & Picófila (< 0.2 cm) \\
\hline & & le & Leptófila (0.2cm-0.8cm) \\
\hline & & na & Nanófila (0.8cm-2.5cm) \\
\hline & & mi & Micrófila (2.5cm-7.5cm) \\
\hline & & no & Notófila (7.5cm-12.5cm) \\
\hline & & me & Mesófila (12.5cm-25cm) \\
\hline & & pl & Platífila $(25 \mathrm{~cm}-36 \mathrm{~cm})$ \\
\hline & & $m a$ & Macrófila $(36$ cm-83cm) \\
\hline & & $m g$ & Megáfila (> 83 cm) \\
\hline & \multirow{4}{*}{ Inclinación foliar } & $v e$ & Vertical (> $30^{\circ}$ por encima de la horizontal) \\
\hline & & la & Lateral ( $\pm 30^{\circ}$ a la horizontal) \\
\hline & & pe & Pendular (> 30 por debajo de la horizontal) \\
\hline & & co & Compuesta \\
\hline & \multirow{7}{*}{ Clorotipo foliar } & do & Dorsoventral \\
\hline & & is & Isobilateral o isocéntrico \\
\hline & & de & Caducifolio \\
\hline & & $c t$ & Córtico (tallo fotosintético) \\
\hline & & $a c$ & Aclorófilo (sin clorofila) \\
\hline & & ro & Roseta \\
\hline & & so & Sólido tridimensional \\
\hline & \multirow{4}{*}{ Morfotipo foliar } & su & Suculento \\
\hline & & $p v$ & Nervadura paralela \\
\hline & & fi & Filicoide (helechos) \\
\hline \multirow{12}{*}{$\begin{array}{l}\text { Estructura vascular de } \\
\text { soporte }\end{array}$} & & $c a$ & Insectívoro \\
\hline & \multirow{6}{*}{ Forma de vida } & ph & Fanerofita \\
\hline & & $c h$ & Caméfita \\
\hline & & $h c$ & Hemicriptófita \\
\hline & & $\mathrm{cr}$ & Criptófita \\
\hline & & th & Terófita \\
\hline & & li & Lianoide \\
\hline & \multirow{5}{*}{ Tipo de raíz } & ad & Adventicia (e.g. contrafuertes) \\
\hline & & $a e$ & Aérea (e.g. neumatóforos) \\
\hline & & ep & Epifítica (e.g. Tillandsia spp.) \\
\hline & & hy & Hidrofítica (e.g. Nymphaea spp.) \\
\hline & & $p a$ & Parasítica (e.g. Cuscuta spp.) \\
\hline
\end{tabular}




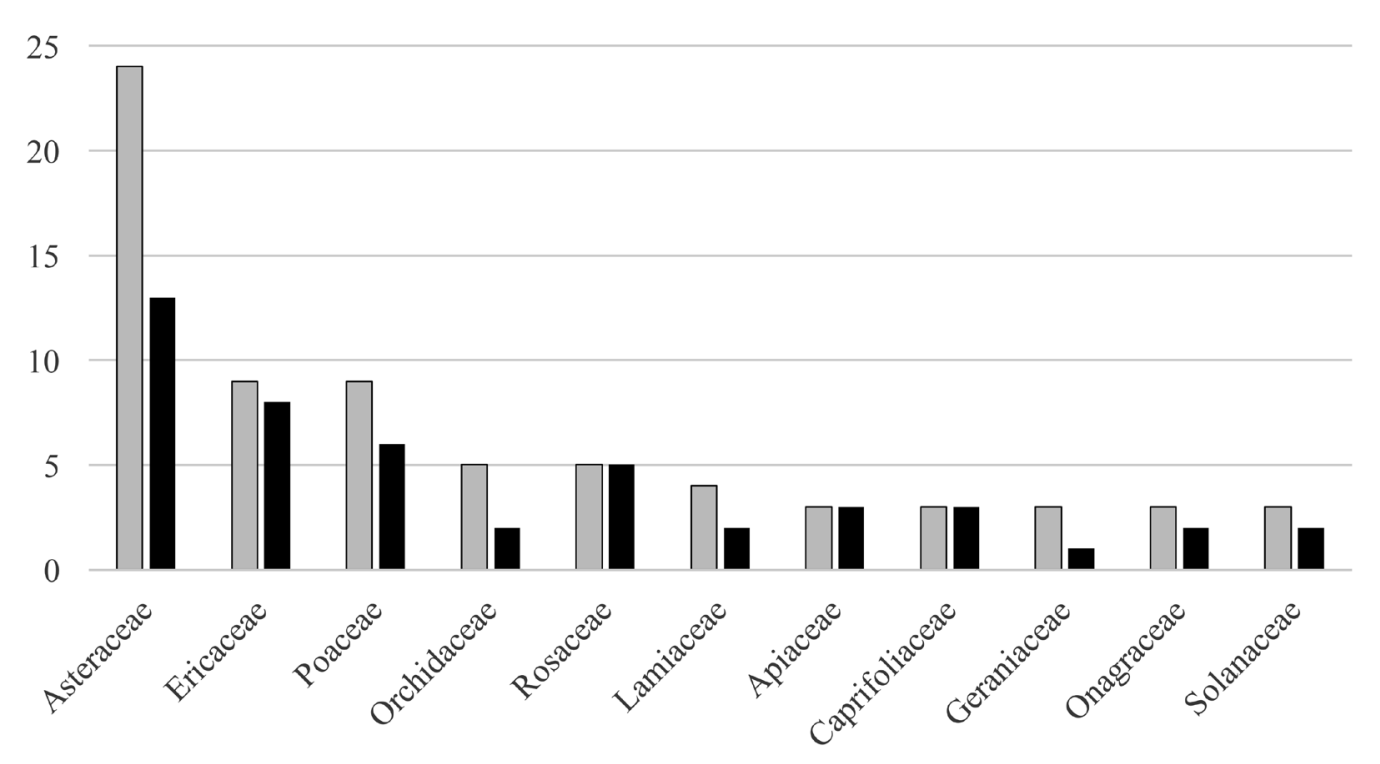

$\square$ Especies -Géneros

Figura 5. Número de especies y géneros de las familias de plantas vasculares mejor representadas en los sitios de bosque de oyamel muestreados.

TABLA 3. Ajuste del modelo logarítmico y potencial para la relación riqueza de especies-área (S-A).

\begin{tabular}{|c|c|c|c|c|c|c|c|c|c|c|c|}
\hline \multirow{2}{*}{ Sitio } & \multicolumn{4}{|c|}{$\begin{array}{c}\text { Modelo logarítmico para S } \\
\text { (ecuación 2) }\end{array}$} & \multicolumn{7}{|c|}{$\begin{array}{c}\text { Modelo potencial para S } \\
\text { (ecuación 1) }\end{array}$} \\
\hline & $c$ & $z$ & $R^{2}$ & RECM & $c$ & $z$ & $\begin{array}{l}R^{2} \\
\text { aritmético }\end{array}$ & $F C$ & $E E R$ & RECM & $\begin{array}{l}R^{2} \\
\log -\log \end{array}$ \\
\hline 1 & -1.4729 & 3.3697 & 0.9925 & 0.4336 & 3.8665 & 0.2620 & 0.9572 & 1.0043 & 0.0929 & 1.1532 & 0.9667 \\
\hline 2 & 1.0071 & 4.3802 & 0.9867 & 0.7530 & 7.2689 & 0.2195 & 0.9691 & 1.0027 & 0.0732 & 1.2247 & 0.9704 \\
\hline 3 & 4.5248 & 2.7385 & 0.9201 & 1.1940 & 7.8604 & 0.1621 & 0.9181 & 1.0036 & 0.0843 & 1.2191 & 0.9309 \\
\hline 4 & 2.0475 & 4.2538 & 0.9650 & 1.1982 & 8.3924 & 0.1961 & 0.9960 & 1.0003 & 0.0247 & 0.4196 & 0.9957 \\
\hline 5 & 7.5666 & 3.5592 & 0.9818 & 0.7162 & 11.3120 & 0.1562 & 0.9519 & 1.0020 & 0.0632 & 1.2146 & 0.9570 \\
\hline 6 & -5.1613 & 4.2950 & 0.8732 & 2.4217 & 3.6575 & 0.2814 & 0.9711 & 1.0045 & 0.0943 & 1.3249 & 0.9701 \\
\hline 7 & -1.2041 & 3.7903 & 0.9248 & 1.5988 & 5.3602 & 0.2281 & 0.9842 & 1.0020 & 0.0633 & 0.7936 & 0.9793 \\
\hline 8 & -0.5420 & 4.5281 & 0.9964 & 0.4054 & 6.3302 & 0.2383 & 0.9722 & 1.0023 & 0.0678 & 1.2287 & 0.9783 \\
\hline 9 & 0.9676 & 4.1906 & 0.9866 & 0.7234 & 7.1699 & 0.2139 & 0.9900 & 1.0008 & 0.0393 & 0.6539 & 0.9908 \\
\hline 10 & -4.3064 & 5.3500 & 0.9950 & 0.5607 & 4.6447 & 0.2973 & 0.9326 & 1.0073 & 0.1207 & 2.3757 & 0.9568 \\
\hline 11 & 3.4218 & 4.6536 & 0.9330 & 1.8442 & 10.3380 & 0.1823 & 0.9824 & 1.0011 & 0.0470 & 1.0111 & 0.9821 \\
\hline 12 & -8.0077 & 5.6858 & 0.9146 & 2.5698 & 3.8550 & 0.3126 & 0.9832 & 1.0048 & 0.0980 & 1.2002 & 0.9738 \\
\hline 13 & -1.7150 & 6.6544 & 0.8716 & 0.9705 & 3.3914 & 0.3436 & 0.9688 & 1.0130 & 0.1610 & 2.0752 & 0.9432 \\
\hline 14 & 6.9544 & 2.3379 & 0.9838 & 0.4433 & 9.3392 & 0.1343 & 0.9763 & 1.0006 & 0.0351 & 0.5421 & 0.9817 \\
\hline 15 & -3.5622 & 4.2952 & 0.9144 & 1.9446 & 4.5305 & 0.2604 & 0.9847 & 1.0013 & 0.0519 & 0.8973 & 0.9892 \\
\hline Promedio & & & 0.9493 & 1.1852 & & & 0.9692 & & & 1.1556 & 0.9711 \\
\hline
\end{tabular}


TABLA 4. Ajuste del modelo logarítmico y potencial para la relación riqueza de tipos funcionales área $\left(\mathrm{S}_{\mathrm{g}}-\mathrm{A}\right)$

\begin{tabular}{|c|c|c|c|c|c|c|c|c|c|c|c|}
\hline \multirow{2}{*}{ Sitio } & \multicolumn{4}{|c|}{$\begin{array}{c}\text { Modelo logarítmico para } \\
S_{g} \text { (ecuación 2) }\end{array}$} & \multicolumn{7}{|c|}{$\begin{array}{c}\text { Modelo potencial para } \\
\left.S_{g} \text { (ecuación } 1\right)\end{array}$} \\
\hline & $c$ & $z$ & $R^{2}$ & RECM & $c$ & $z$ & $\begin{array}{l}R^{2} \\
\text { aritmético }\end{array}$ & $F C$ & $E E R$ & RECM & $\begin{array}{l}R^{2} \\
\log -\log \end{array}$ \\
\hline 1 & 1.4341 & 2.0854 & 0.9518 & 0.6941 & 3.8926 & 0.2129 & 0.8683 & 1.0089 & 0.1333 & 1.2496 & 0.9030 \\
\hline 2 & 5.4341 & 2.0854 & 0.9518 & 0.6941 & 7.3401 & 0.1491 & 0.8972 & 1.0037 & 0.0855 & 1.0600 & 0.9173 \\
\hline 3 & 5.6366 & 1.4533 & 0.8890 & 0.7598 & 7.1506 & 0.1149 & 0.9198 & 1.0025 & 0.0700 & 0.6523 & 0.9077 \\
\hline 4 & 5.3681 & 2.2958 & 0.9945 & 0.2533 & 7.7834 & 0.1496 & 0.9713 & 1.0010 & 0.0442 & 0.6005 & 0.9767 \\
\hline 5 & 6.2648 & 2.3162 & 0.9469 & 0.8114 & 8.6793 & 0.1398 & 0.9439 & 1.0019 & 0.0615 & 0.8381 & 0.9496 \\
\hline 6 & 1.2552 & 2.0417 & 0.7454 & 1.7652 & 4.6466 & 0.1725 & 0.8395 & 1.0110 & 0.1478 & 1.4885 & 0.8324 \\
\hline 7 & 1.4480 & 2.3589 & 0.9636 & 0.6787 & 4.8349 & 0.1934 & 0.9777 & 1.0014 & 0.0526 & 0.5311 & 0.9801 \\
\hline 8 & -0.4964 & 2.8217 & 0.9595 & 0.8567 & 3.9779 & 0.2344 & 0.9669 & 1.0024 & 0.0699 & 0.7854 & 0.9762 \\
\hline 9 & 5.4641 & 2.0006 & 0.9690 & 0.5297 & 7.7141 & 0.1351 & 0.9901 & 1.0003 & 0.0244 & 0.3044 & 0.9911 \\
\hline 10 & 1.7125 & 2.7804 & 0.9527 & 0.9162 & 5.1134 & 0.2133 & 0.8778 & 1.0077 & 0.1241 & 1.5871 & 0.9152 \\
\hline 11 & 5.0740 & 3.2223 & 0.9695 & 0.8455 & 9.1933 & 0.1613 & 0.9899 & 1.0004 & 0.0283 & 0.4903 & 0.9916 \\
\hline 12 & -0.0849 & 2.7799 & 0.9397 & 1.0419 & 4.4517 & 0.2163 & 0.9815 & 1.0020 & 0.0629 & 0.5942 & 0.9773 \\
\hline 13 & -0.5251 & 3.2221 & 0.9252 & 1.3554 & 4.8889 & 0.2210 & 0.9759 & 1.0031 & 0.0791 & 0.8031 & 0.9661 \\
\hline 14 & 6.1164 & 1.8328 & 0.9876 & 0.3031 & 7.9766 & 0.1265 & 0.9930 & 1.0002 & 0.0192 & 0.2280 & 0.9937 \\
\hline 15 & -0.9527 & 2.9907 & 0.9907 & 0.4288 & 3.8659 & 0.2450 & 0.9843 & 1.0011 & 0.0469 & 0.5997 & 0.9900 \\
\hline Promedio & & & 0.9425 & 0.7956 & & & 0.9451 & & 0.0700 & 0.7875 & 0.9512 \\
\hline
\end{tabular}

La figura 6 presenta las relaciones entre los parámetros del modelo potencial (Tabla 3 y 4), mostrando que el ajuste estadístico $\left(\mathrm{R}^{2}\right)$ es relativamente bajo, particularmente entre los parámetros de la relación $\mathrm{S}_{\mathrm{g}}$-A. La dispersión de los parámetros sobre la relación teórica entre ellos implica que las líneas rectas (espacio log-log) se intersectan en una zona amplia, sin converger en un punto común o zona reducida de intersección, de modo que las relaciones entre los parámetros tienen alta incertidumbre para su uso en estimaciones.

En el caso de la relación $S-S_{g}$, se estimó con la ecuación (43) ajustada directamente por regresión estadística de los datos muestrales (todas las áreas). La figura 7 muestra el ajuste estadístico con una baja dispersión, implicando una zona compacta de intersección, por lo que la ecuación (18) puede considerarse con baja incertidumbre. Asimismo, se muestra la misma relación $S-S_{g}$ pero producto de aplicar la ecuación (15) usando los parámetros c, $\mathrm{c}_{\mathrm{g}}$, z y z $\mathrm{z}_{\mathrm{g}}$ estimados de las relaciones $\mathrm{S}-\mathrm{A}$ y $\mathrm{S}_{\mathrm{g}}$-A ajustadas (Tabla 3 y 4). Ambos tipos de ajustes resultaron en metaparámetros $\left(\mathrm{a}_{\mathrm{sg}} \mathrm{y} \mathrm{b}_{\mathrm{sg}}\right)$ comparables y con ajustes similares $\left(\mathrm{R}^{2}\right)$. Lo anterior muestra que los parámetros de la relación $\mathrm{S}-\mathrm{S}_{\mathrm{g}}$ es robusta y puede obtenerse directamente de los parámetros de las curvas riqueza-área.

La aplicación del modelo logarítmico a las curvas S-A y $\mathrm{S}_{\mathrm{g}}$-A se evaluó mediante la relación $\mathrm{S}$ - $\mathrm{S}_{\mathrm{g}}$ dada por la ecuación (35), que es un modelo lineal con una constante aditiva y una multiplicativa. La figura 8 ilustra la relación entre los parámetros de S-S $S_{g}$, donde el ajuste del patrón de la ecuación (35) indica mayor dispersión para el caso de los parámetros de la relación S-S del modelo potencial (Fig. 7).

El uso de las relaciones A-S y A-S ${ }_{\mathrm{g}}$, en las ecuaciones (23) y (24), para estimar los parámetros de $S-S_{g}$, ecuación (25), resultó en un mejor ajuste (Fig. 9) que en el caso de las relaciones S-A y $\mathrm{S}_{\mathrm{g}}$-A (Fig. 7), en comparación con el 
caso del ajuste directo por regresión lineal del patrón de los parámetros S-S (Fig. 7).

La figura 10 muestra los patrones de los parámetros de las relaciones A-S y A-S , ecuaciones (23) y (24), donde resultan mejores ajustes que en el caso de los patrones $\mathrm{S}-\mathrm{A}$ y $S_{g}$-A (Fig. 6).

Se puede estimar $\mathrm{S}$ para un valor dado de $\mathrm{S}_{\mathrm{g}}$ asociado a un área de muestreo, mediante la relación $\mathrm{S}-\mathrm{S}_{\mathrm{g}}$ (áreas de muestreo implícitas), con valores medidos en campo (Fig. 11), donde la relación entre el valor de $S$ medido y el estimado mediante la relación $S-S_{g}$, muestra un sesgo (constante aditiva de la línea recta de ajuste) relativamente alto. La relación que es forzada a pasar por el origen brinda una visión de la bondad del ajuste (Fig. 11).

La estimación de $\mathrm{S}$ usando la relación $\mathrm{A}-\mathrm{S}$, ecuación (42) para obtener $b_{\text {sg }}$ de la ecuación (47) y utilizar la ecua- ción (45) (Fig. 9), para obtener $\mathrm{a}_{\mathrm{sg}} \mathrm{y}$ así aplicar la ecuación (43) para obtener S (Fig. 12). Los resultados obtenidos usando la relación A-S presentan menor sesgo que los obtenidos con estimaciones directas (Fig. 11).

En el caso de estimar $\mathrm{S}_{\mathrm{g}}$ dado $\mathrm{S}$, usando la relación entre ambas variables se obtiene un sesgo relativamente alto, al igual que el caso anterior (Fig. 13).

La figura 14 muestra la estimación de $\mathrm{S}_{\mathrm{g}}$ usando la relación A-S, con un procedimiento similar al usado para el caso de A- $\mathrm{S}_{\mathrm{g}}$. Los resultados tienen menos sesgo que el caso de las estimaciones directas (Fig. 13).

Las relaciones entre los parámetros de las curvas S-A, $\mathrm{S}_{\mathrm{g}}$-A o $\mathrm{S}-\mathrm{S}_{\mathrm{g}}$, o las funciones inversas, pueden mejorarse usando un factor de escala q, ecuación (12). La figura 15 muestra un ejemplo de la relación de los parámetros de $S_{g}$-A (Fig. 6).
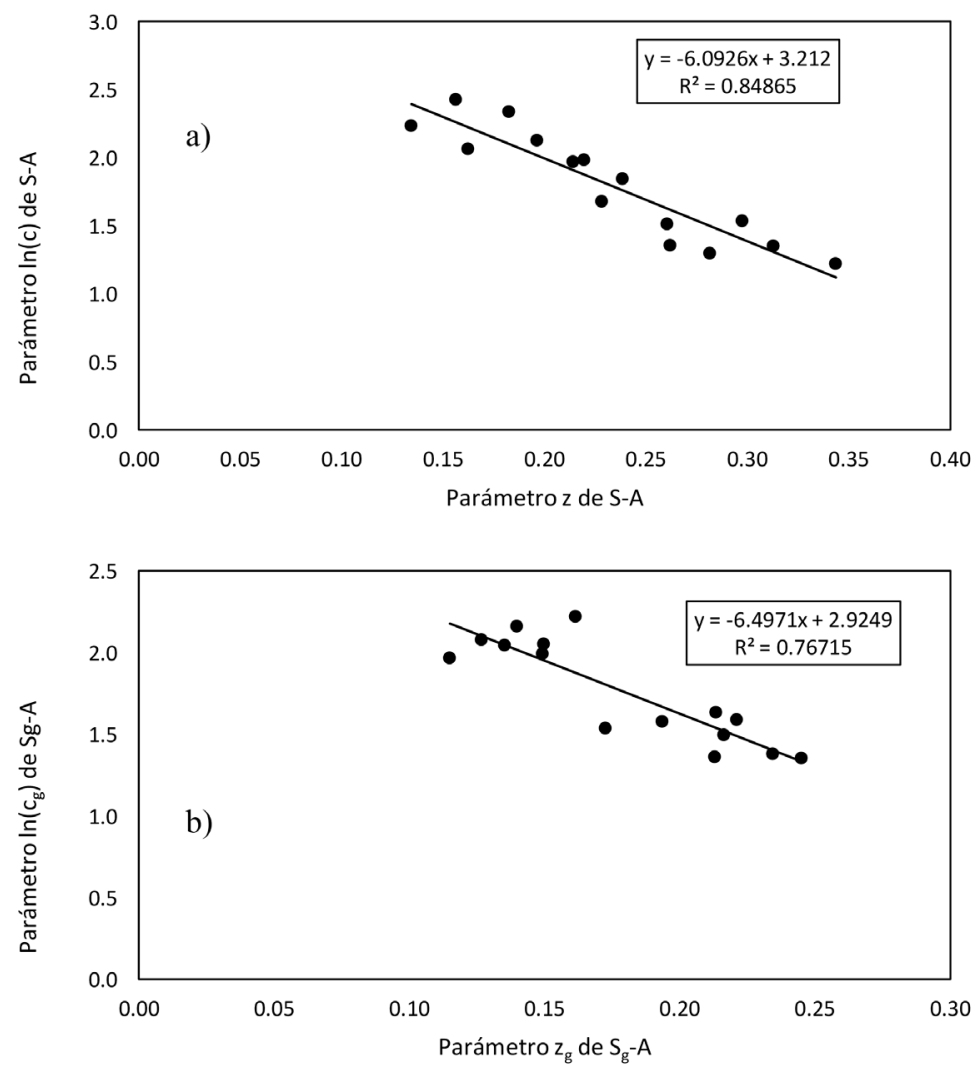

Figura 6. (a) Relación entre los parámetros de las curvas riqueza de especies-área $(\mathrm{S}-\mathrm{A})$ y $(\mathrm{b})$ riqueza de tipos funcionales-área $\left(\mathrm{S}_{\mathrm{g}}-\mathrm{A}\right)$. 

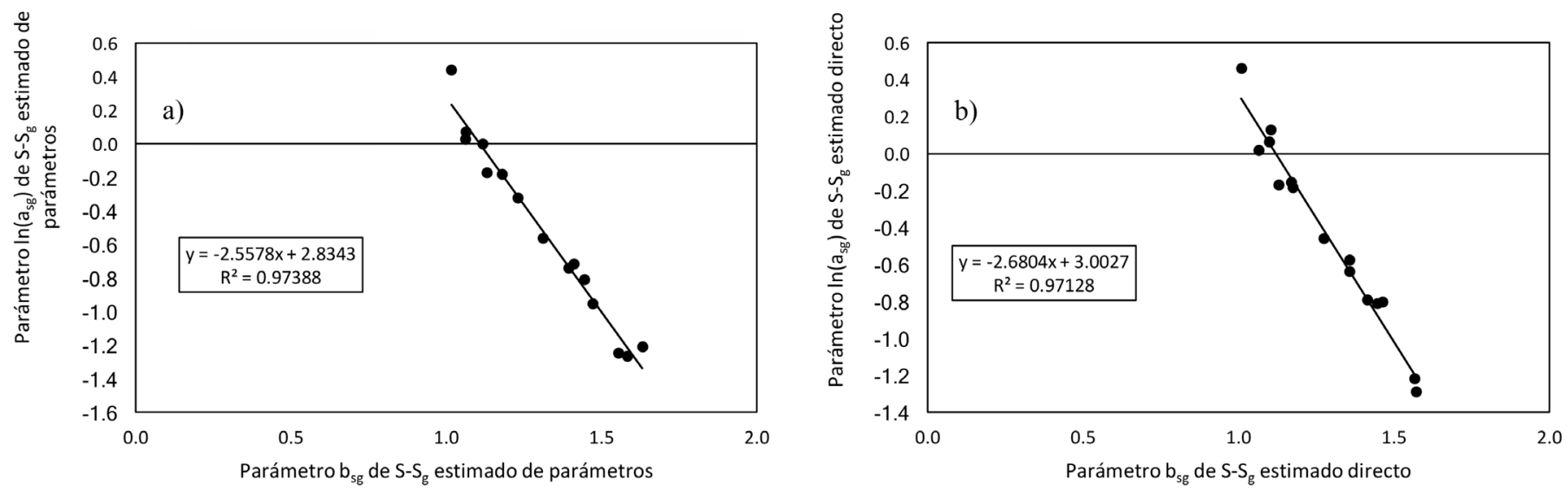

FiguRa 7. Relación entre los parámetros de la relación riqueza de especies-riqueza de tipos funcionales (S-S g $_{\mathrm{g}}$ estimada usando: (a) los parámetros de las relaciones de riqueza-área $\left(\mathrm{S}-\mathrm{A}\right.$ y $\left.\mathrm{S}_{\mathrm{g}}-\mathrm{A}\right)$, y (b) directamente por la regresión estadística entre $\mathrm{S}$ y $\mathrm{S}_{\mathrm{g}}$

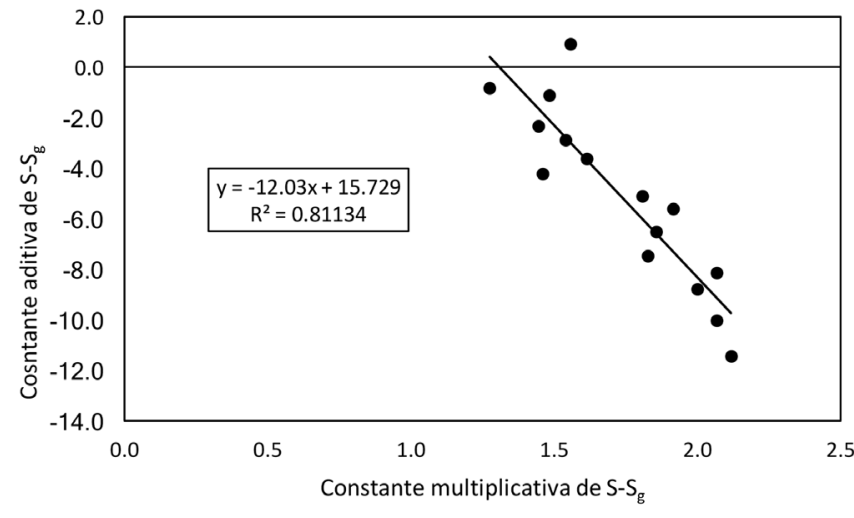

Figura 8. Relación entre la constante multiplicativa y aditiva del modelo logarítmico para la relación riqueza de especiesriqueza de tipos funcionales $\left(\mathrm{S}-\mathrm{S}_{\mathrm{g}}\right)$, ajustado directamente por regresión estadística.

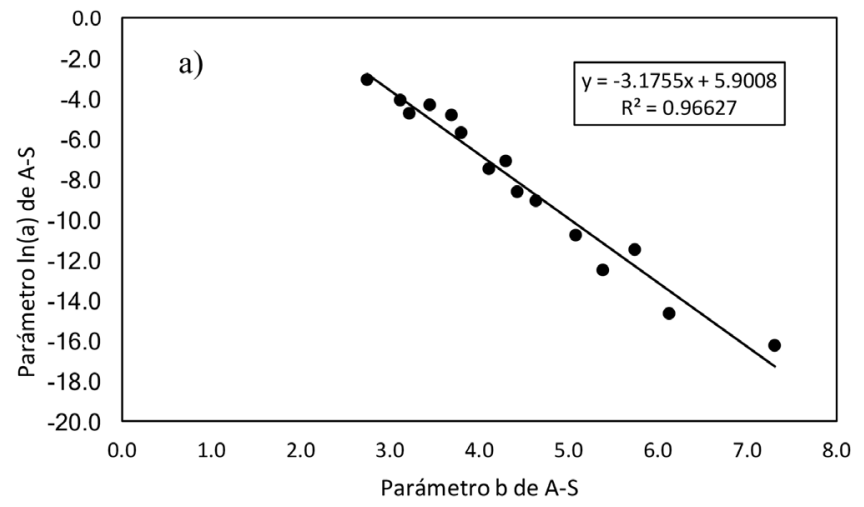

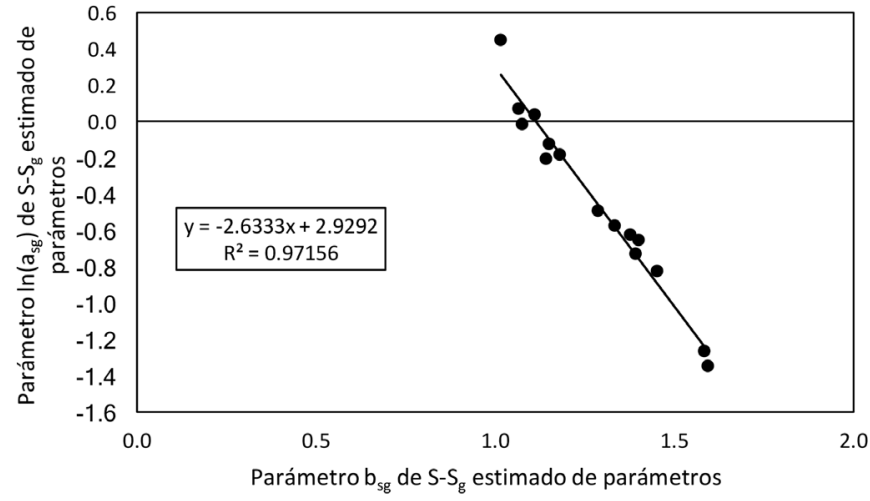

Figura 9. Relación entre los parámetros de la relación riqueza de especies-riqueza de tipos funcionales $\left(\mathrm{S}-\mathrm{S}_{\mathrm{g}}\right)$, estimada usando los parámetros de las relaciones A-S y A-S.

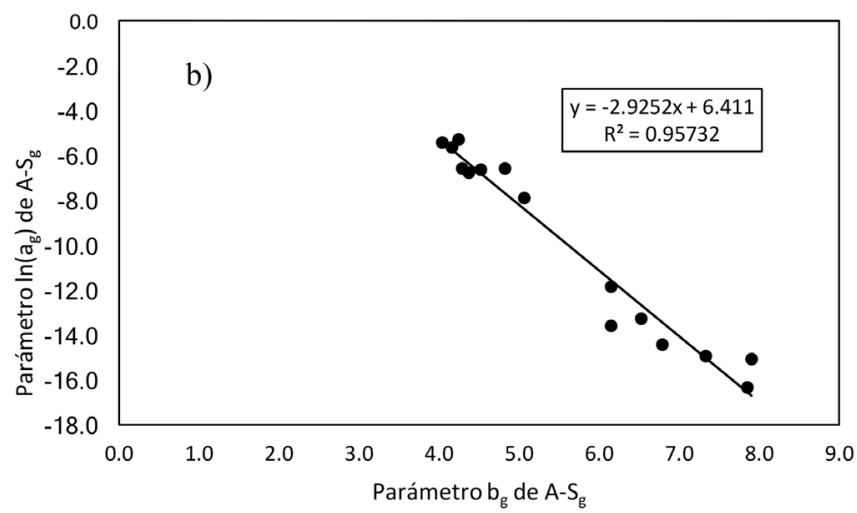

Figura 10. Relación entre los parámetros de la relación del área con (a) riqueza de especies (A-S) y (b) con tipos funcionales (A-S $)$. 

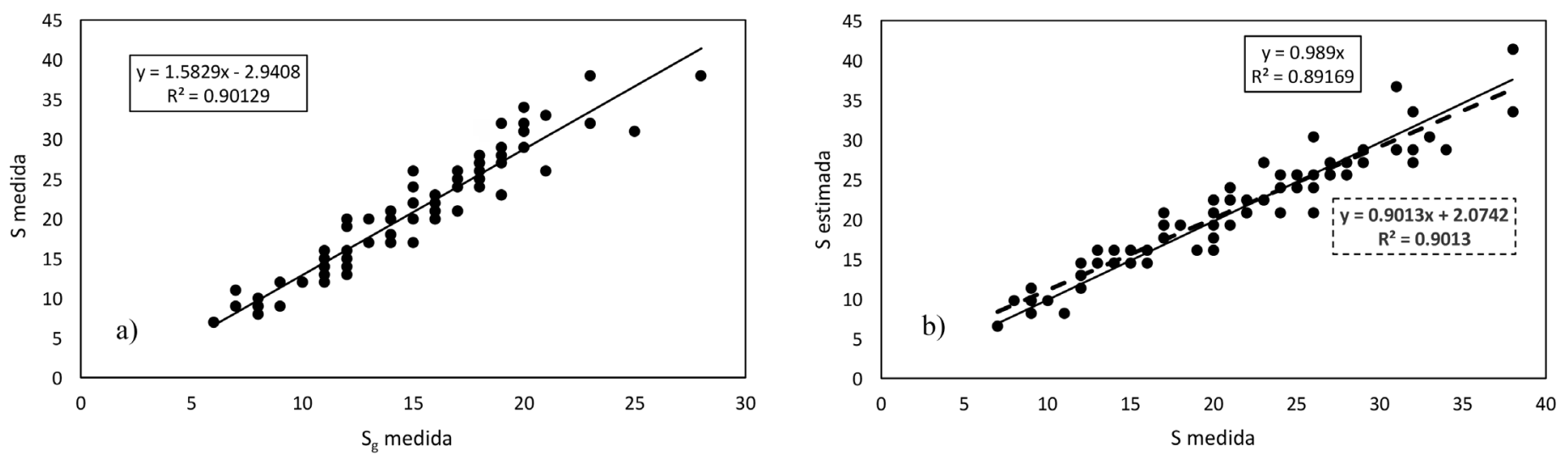

Figura 11. Estimación de riqueza de especies ( $\mathrm{S}$ ) a partir de (a) la riqueza de tipos funcionales $\left(\mathrm{S}_{\mathrm{g}}\right)$, usando la relación entre $\mathrm{S}$ y $\mathrm{S}_{\mathrm{g}}$ y (b) los resultados de las estimaciones.

La línea de ajuste continua representa el caso de la regresión forzada a pasar por el origen y la punteada el caso contrario.

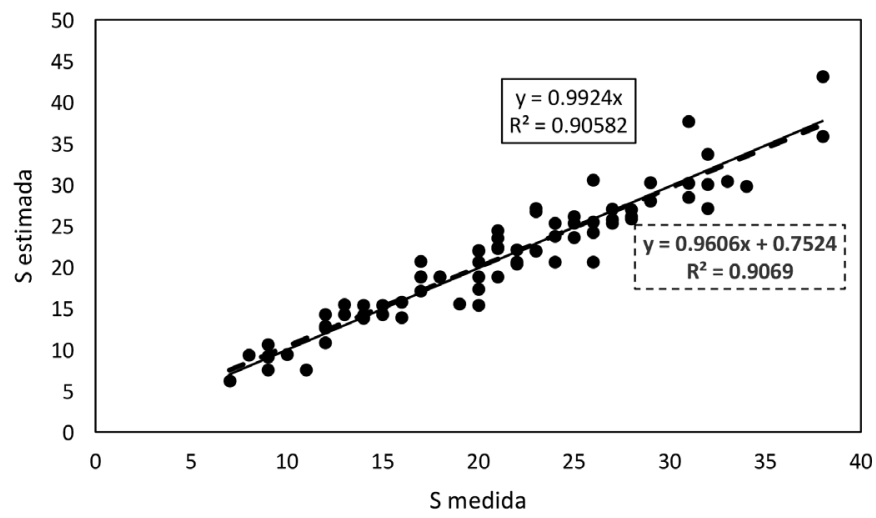

Figura 12. Estimación de $S$ de la relación A-S para estimar el exponente de la relación S-S. La línea de ajuste continua representa el caso de la regresión forzada a pasar por el origen y la punteada el caso contrario.

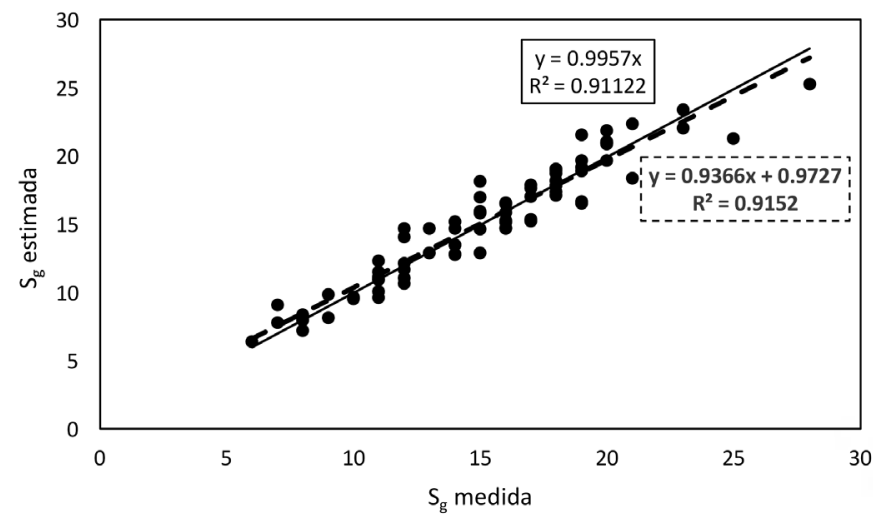

Figura 14. Estimación de Sg de la relación A-S para estimar el exponente de la relación S-Sg. La línea de ajuste continua representa el caso de la regresión forzada a pasar por el origen y la punteada el caso contrario.

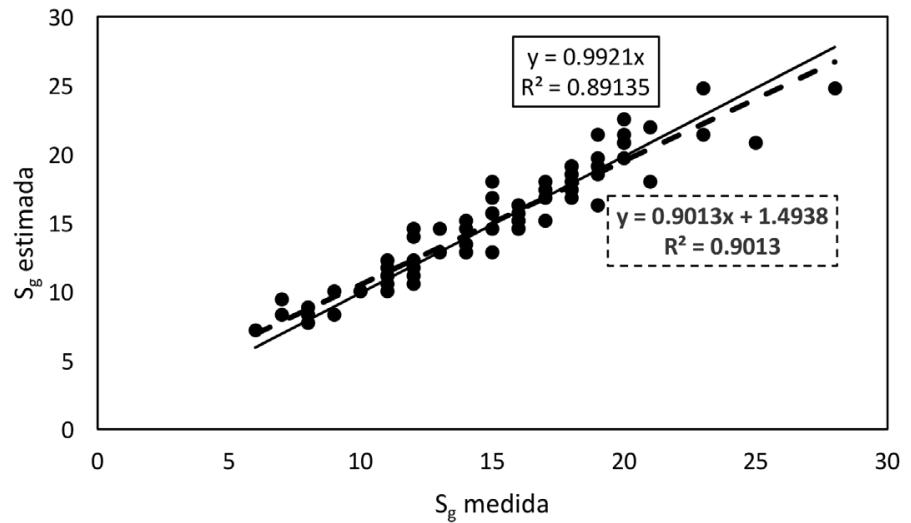

Figura 13. Estimación de $\mathrm{S}_{\mathrm{g}}$ de la relación entre $\mathrm{S}$ y $\mathrm{S}_{\mathrm{g}}$ medidos. La línea de ajuste continua representa el caso de la regresión forzada a pasar por el origen y la punteada el caso contrario.

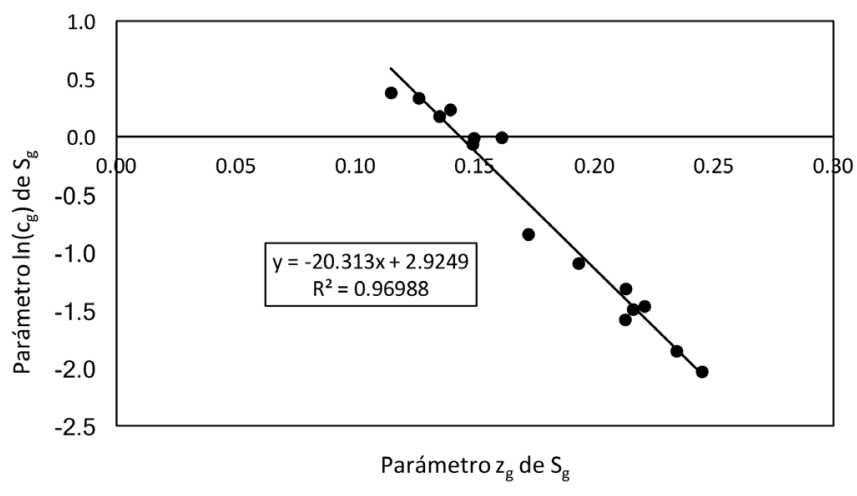

Figura 15. Relación entre los parámetros de $\mathrm{S}_{\mathrm{g}} \mathrm{A}$, con un factor de escala de $\mathrm{q}=1000000$ para las áreas de muestreo. 


\section{DISCUSIÓN}

La interpretación teórica de la relación riqueza de especies-área se ha enfocado en diferentes hipótesis (Connor y McCoy, 1979; McGuinness, 1984; Turner y Tjørve, 2005), sin obtener evidencia contundente de su validez generalizada (Connor y McCoy, 1979). Aunque el problema teórico de la comprensión de la relación entre riqueza y área permanece, las relaciones empíricas desarrolladas tienen limitaciones en la parte de los ajustes estadísticos y en la complejidad de hacer operativos sus resultados (Wright, 1981; McGuinness, 1984).

Los resultados obtenidos al usar las relaciones entre el número de tipos funcionales y el de especies vasculares podrían permitir métodos alternativos para obtener indicadores de diversidad (Gillison et al., 2003 y 2013; Gillison y Liswanti, 2004), dado que la estimación de la riqueza de tipos funcionales tiene requerimientos mínimos de entrenamiento profesional, haciendo el uso de $\mathrm{S}_{\mathrm{g}}$ costo-efectivo para enfoques de inventarios comunitarios o similares.

Desde un enfoque estrictamente matemático, independientemente de su interpretación ecológica, el uso de los patrones entre los parámetros de las relaciones funcionales $\mathrm{S}-\mathrm{A}, \mathrm{S}_{\mathrm{g}}-\mathrm{A}$ y $\mathrm{S}-\mathrm{S}_{\mathrm{g}}$, y sus relaciones inversas, permite simplificar el problema de estimación conjunta de $S$ y $S_{g}$. Los resultados obtenidos muestran que el enfoque utilizado permite estimaciones comparables, o mejores, que los ejercicios directos de medición.

Un problema asociado a las estimaciones directas de $S$ dado $S_{g}$, o de $S_{g}$ dado $S$, es el requerimiento de generalización en su aplicabilidad en un tipo de vegetación o ecosistema. La figura 15 muestra que, al elegir unidades de medida adecuadas (factores de escala), es posible utilizar el marco teórico presentado en forma robusta y con reducción de incertidumbres importantes, haciendo uso de “artefactos" del método, a favor del investigador.

La propuesta de la relación entre los exponentes de las relaciones A-S, A-S y S-S permite la simplificación de estimaciones de la riqueza estableciendo un vínculo entre sus exponentes, de manera que, con el conocimiento de una de ellas, el resto pueden ser calculadas directamente.

\section{CONCLUSIONES}

El marco teórico presentado permite relacionar los parámetros de las curvas de riqueza y áreas de muestreo (anidadas) con base en las propiedades matemáticas de un conjunto de rectas que se interceptan en una zona común. Este análisis generó estimaciones de riqueza de especies equiparables con las mediciones directas, a partir del número de tipos funcionales, caracterizados con un método que no requiere conocimientos taxonómicos.

Los hallazgos obtenidos pueden simplificar considerablemente la obtención de indicadores de diversidad en inventarios de plantas vasculares. Sin embargo, los alcances de esta investigación están orientados a la vegetación sucesionalmente madura, por lo tanto, su aplicación en bosques bajo disturbio o en otras etapas sucesionales, requerirá de evidencia experimental adicional, particularmente para la relación entre la riqueza de plantas vasculares y otros taxa de interés.

\section{REFERENCIAS}

Arrhenius, O. (1921). Species and area. Journal of Ecology, 9(1), 95-99. doi:10.2307/2255763

Baskerville, G. L. (1972). Use of logarithmic regression in the estimation of plant biomass. Canadian Journal of Forestry, 2(1), 49-53.

Brooks, T. M., Mittermeier, R. A., Mittermeier, C. G., daFonseca, G. A. B., Rylands, A. B., Konstant, W. R., Flick, P., Pilgrim, J., Oldfield, S., Magin, G., \& Hilton-Taylor, G. (2001). Habitat loss and extinction in the hotspots of biodiversity. Conservation Biology, 16, 909-923.

Byng, J., Chase, M., Christenhusz, M., Fay, M., Judd, W., Mabberley, D., Sennikov, A., Soltis, D., Soltis, P., \& Stevens, P. (2016). An update of the Angiosperm Phylogeny Group classification for the orders and families of flowering plants: APG IV. Botanical Journal of the Linnean Society, 181(1), 1-20. doi:10.1111/boj.12385

Connor, E. F., \& McCoy, E. D. (1979). The statistics and biology of the species-area relationship. The American Naturalist, 113(6), 791-833. 
Convention on Biological Diversity [CBD]. (2010). Global Biodiversity Outlook 3. Montreal, Quebec, Canada: Secretariat of the Convention on Biological Diversity.

Cooper, H. D., \& Noonan-Mooney, K. (2013). Convention on Biological Diversity. In S. A., Levin (Ed.), Encyclopedia of Biodiversity (2nd ed.) (pp. 306-319). Waltham, MA: Academic Press.

Cresswell, J. E., Vidal-Martinez, V. M., \& Crichton, N. J. (1995). The investigation of saturation in the species richness of communities: some comments on methodology. Oikos, 72(2), 301-304.

Croezen, H., Bergsma, G., Clemens, A., Sevenster, M., \& Tulleners, B. (2011). Biodiversity and land use. A search for suitable indicators for policy use. Delft: CE Delft.

Dengler, J. (2008). Pitfalls in Small-Scale Species-Area Sampling and Analysis. Folia Geobotanica, 43, 269-287. doi:10.1007/s12224-008-9014-9

Dengler, J. (2009). Which function describes the species-area relationship best? A review and empirical evaluation. Journal of Biogeography, 36: 728-744. doi:10.1111/j.1365-2699.2008.02038.x

Díaz, S., \& Cabido, M. (2001). Vive la difference: plant functional diversity matters to ecosystem processes. Trends in Ecology \& Evolution, 16(11), 646-655.

Dobson, A. (2005). Monitoring global rates of biodiversity change: challenges that arise in meeting the Convention on Biological Diversity (CBD) 2010 goals. Philosophical Transactions of the Royal Society B-Biological Sciences, 360(1454): 229-241.

Drakare, S., Lennon, J. L., \& Hillebrand, H. (2006). The imprint of the geographical, evolutionary and ecological context on species-area relationships. Ecology Letters, 9(2), 215-227.

Dumortier, M., Butaye, J., Jacquemyn, H., Van-Camp, N., Lust, N., \& Hermy, M. (2002). Predicting vascular plant species richness of fragmented forests in agricultural landscapes in central Belgium. Forest Ecology and Management, 158(1-3), 85-102.

Franklin, J. F. (1988) Structural and functional diversity in temperate forests. In E. O. Wilson, \& F. M. Peter (Eds.) Bio- diversity (pp. 166-175). Washington, D.C.: National Academy Press.

Gao, T., Nielsen, A. B., \& M. Hedblom. (2015). Reviewing the strength of evidence of biodiversity indicators for forest ecosystems in Europe. Ecological Indicators, 57, 420434. doi:10.1016/j.ecolind.2015.05.028

Gillison A. N., Bignell, D. E., Brewer, K. R. W., Fernandes, E. C. M., Jones, D. T., Sheiz, D., May, P. H., Watt, A. D., Constantino, R., Couto, E. G., Hairiah, K., Jepson, P., Kartono, A. P., Maryauto, I., Neto, G. G., van Noordwisk, M., Silveira, E. A., Susilo, F. X., Vosti, S. A., \& Nunes, P. C. (2013). Plant functional types and traits as biodiversity indicators for tropical forests: two biogeographically separated case studies including birds, mammals and termites. Biodiversity and Conservation, 22(9), 1909-1930. doi:10.1007/s10531-013-0517-1

Gillison, A. N. (1981). Towards a functional vegetation classification. In A. N. Gillison, \& D. J. Anderson (Eds). Vegetation classification in Australia (pp. 30-41). Canberra, Australia: CSIRO and Australian National University Press.

Gillison, A. N. (2002). A generic, computer assisted method for rapid vegetation classification and survey: tropical and temperate case studies. Conservation Ecology, 6(2), 1-17.

Gillison, A. N. (2006). A field manual for rapid vegetation classification and survey for general purposes. Jakarta, Indonesia: Center for International Forestry Research.

Gillison, A. N. (2016). Vegetation functional types and traits at multiple scales. In E. O. Box (Ed.), Vegetation Structure and Function at Multiple Spatial, Temporal and Conceptual Scales, Geobotany Studies (pp. 53-97). Switzerland: Springer International Publishing.

Gillison, A. N., \& Carpenter, G. (1997). A generic plant functional attribute set and grammar for dynamic vegetation description and analysis. Functional Biology, 11, 775783.

Gillison, A. N., \& Liswanti, N. (2004). Assessing biodiversity al landscape level in northern Thailand and Sumatra (Indonesia): the importance of environmental context. Agriculture Ecosystems \& Environment, 104(1), 75-86. 
Gillison, A. N., Jones, D. T., Susilo, F. X., \& Bignell, D. E. (2003). Vegetation indicates diversity of soil macroinvertebrates: a case study with termites along land-use intensification gradient in lowland Sumatra. Organisms Diversity \& Evolution, 3(2), 111-126.

Gitay, H., \& Noble, I. R. (1997). What are plant functional types and how should we seek them? In T. M. Smith, H. H. Shugart, \& F. I. Woodward (Eds.), Plant Functional Types (pp. 3-19). Cambridge, UK: Cambridge University Press.

Gleason, H. A. (1922). On the relationship between species and area. Ecology, 3(2), 158-162. doi:10.2307/1929150

Gould, S. J. (1979). An allometric interpretation of species-area curves: the meaning of the coefficient. The American Naturalist, 114(3), 335-343.

Hooper, D. U., Chapin III, F. S., Ewel, J. J., Hector, A., Inchausti, P., Lavorel, S., Lawton, J. H., Lodge, D. M., Loreau, M., Naeem, S., Schmid, B., Setälä, H., Symstad, A. J., Vandermeer, J., \& Wardle, D. A. (2005). Effects of biodiversity on ecosystem functioning: a consensus of current knowledge. Ecological Monographs, 75(1), 3-35. doi:10.1890/04-0922

Hopkins, B. (1955). The species-area relations of plant communities. Journal of Ecology, 43, 409-426.

Koh, L. P., \& Ghazoul, J. A. 2010. Matrix-calibrated speciesarea model for predicting biodiversity losses due to landuse change. Conservation Biology, 24(4), 994-1001.

Lavorel, S., McIntyre, S., Landsberg, J., \& Forbes, T. D. A. (1997). Plant functional classifications: from general groups to specific groups based on response to disturbance. Trends in Ecology \& Evolution, 12(12), 474-478. doi:10.1016/s0169-5347(97)01219-6

Lawton, J. H. (1999). Are there general laws in ecology? Oikos, $84(2), 177-192$.

Lewandowski, A. S., Noss, R. F., \& Parsons, D. R. (2010). The Effectiveness of Surrogate Taxa for the Representation of Biodiversity. Conservation Biology, 24(5), 13671377. doi:10.1111/j.1523-1739.2010.01513.x

Liang, J., Crowther, T. W., Picard, N., Wiser, S., Zhou, M., Alberti, G., Schulze, E., McGuire, A. D., Bozzato, F., Pretzsch, H., de Miguel, S., Paquette, A., Hérault, B.,
Scherer-Lorenzen, M., Barrett, C. B., Glick, H. B., Hengeveld, G. M., Nabuurs, G., Pfautsch, S., Viana, H., Vibrans, A. C., Ammer, C., Schall, P., Verbyla, D., Tchebakova, N., Fischer, M., Watson, J. V., Chen, H. Y. H., Lei, X., Schelhaas, M., Lu, H., Gianelle, D., Parfenova, E. I., Salas, C., Lee, E., Lee, B., Kim, H. S., Bruelheide, H., Coomes, D. A., Piotto, D., Sunderland, T., Schmid, B., Gourlet-Fleury, S., Sonké, B., Tavani, R., Zhu, J., Brandl, S., Vayreda, J., Kitahara, F., Searle, E. B., Neldner, V. J., Ngugi, M. R., Baraloto, C., Frizzera, L., Bałazy, R., Oleksyn, J., Zawiła-Niedźwiecki, T., Bouriaud, O., Bussotti, F., Finér, L., Jaroszewicz, B., Jucker, T., Valladares, F., Jagodzinski, A. M., Peri, P. L., Gonmadje, C., Marthy, W., O’Brien, T., Martin, E. H., Marshall, A. R., Rovero, F., Bitariho, R., Niklaus, P. A., Alvarez-Loayza, P., Chamuya, N., Valencia, R., Mortier, F., Wortel, V., Engone-Obiang, N. L., Ferreira, L. V., Odeke, D. E., Vasquez, R. M., Lewis, S. L., \& Reich, P. B. (2016). Positive biodiversity-productivity relationship predominant in global forests. Science, 354(6309): 196-208. doi: 10.1126/ science.aaf8957

Lindenmayer, D. B., \& Likens, G. E. 2011. Direct Measurement Versus Surrogate Indicator Species for Evaluating Environmental Change and Biodiversity Loss. Ecosystems, 14, 47-59. doi:10.1007/s10021-010-9394-6

Loehle, C. (1990). Proper statistical treatment of species-area data. Oikos, 57(1), 143-145.

Lomolino, M. V. (2000). Ecology's most general, yet protean pattern: the species-area relationship. Journal of Biogeography, 27(1), 17-26.

Lomolino, M. V. (2001). The species-area relationship: new challenges for an old pattern. Progress in Physical Geography, 25(1), 1-21.

Lot, A., \& Chiang, F. (Eds.). (1986). Manual de herbario: Administración y manejo de colecciones, técnicas de recolección y preparación de ejemplares botánicos. D.F., México: Consejo Nacional de Flora de México.

Lumer, H. (1936). The relation between $b$ and $k$ in systems of relative growth functions of the form $\mathrm{Y}=\mathrm{bX}^{\mathrm{k}}$. The American Naturalist, 70(727), 188-191. 
Lumer, H., Anderson, B. G., \& Hersh, A. H. (1942). On the significance of the constant $\mathrm{b}$ in the law of allometry $\mathrm{Y}=\mathrm{bx}^{\mathrm{k}}$. American Naturalist, 76, 364-375. doi: $10.1086 / 281053$

MacArthur, R. H., \& Wilson, E.O. (1967). The Theory of Island Biogeography. Princenton, N. J. Princeton University Press.

Mace, G. M., \& Baillie, J. E. M. (2007). The 2010 biodiversity indicators: challenges for science and policy. Conservation Biology, 21(6), 1406-1413.

McGuinness, K. (1984). Equations and explanations in the study of species-area curves. Biological. Reviews, 59, 423-440. doi:10.1111/j.1469-185X.1984.tb00711.x

Missouri Botanical Garden [MBG] (2018). Herbario virtual del Jardín Botánico de Missouri. Recuperado de: http://www. tropicos.org.

National Research Council [NRC]. 2000. Ecological Indicators for the Nation. Washington, DC: The National Academies Press. doi:10.17226/9720.

Newton, A. C., \& Kapos, V. (2002). Biodiversity indicators in national forest inventories. Recuperado de: http://www. fa o.org/forestry/3946-0 e 7 f 052 e e b 66d8935170504e3d01ab348.pdf

Noss, R. F. (1990). Indicators for Monitoring Biodiversity: A Hierarchical Approach. Conservation Biology, 4(4), $355-$ 364. doi:10.1111/j.1523-1739.1990.tb00309.x.

Ohlemüller, R., Bannister, P., Dickinson, K. J. M., Walker, S., Anderson, B. J., \& Wilson, J. B. (2004). Correlates of vascular plant species richness in fragmented indigenous forests: assessing the role of local and regional factors. Community Ecology, 5(1), 45-54. doi:10.1556/ ComEc.5.2004.1.5

Palmer, M. W., McGlinn, D. J., \& Fridley, J. D. (2008). Artifacts and Artifictions in Biodiversity Research. Folia Geobotanica, 43(3), 245-257.doi:10.1007/s12224-008-9012-y

Paz-Pellat, F., Odi-Lara, M., Cano-González, A., BolañosGonzález, M. A., \& Zarco-Hidalgo, A. (2009). Equivalencia ambiental en la productividad de la vegetación. Agrociencia, 43(6), 635-648.

Pearson, T. R. H., Brown, S. L., \& Birdsey, R. A. (2007). Measurement guidelines for the sequestration of forest car- bon. Gen. Tech. Rep. NRS-18. Newtown Square, PA: U.S. Department of Agriculture, Forest Service, Northern Research Station.

Pereira, H. M., \& Daily, G. C. (2006). Modeling biodiversity dynamics in countryside landscapes. Ecology, 87(8), 1877-1885. doi:10.1890/0012-9658(2006)87[1877:mbdicl]2.0.co;2

Phillips, O. L., Hall, P., Gentry, A. H., Sawyer, S. A., \& Vásquez, R. (1994). Dynamics and species richness of tropical rain forests. Proceedings of the National Academy of Sciences, 91(7), 2805-2809. doi:10.1073/pnas.91.7.2805

Preston, F. W. (1962). The canonical distribution of commonness and rarity. Ecology, 43(2), 185-215.

Price, C. A., Enquist, B. J., \& Savage, V. M. (2007). A general model for allometric covariation in botanical form and function. Proceedings of the National Academy of Sciences, 104(32), 13204-13209. doi:10.1073/ pnas.0702242104

Programa Mexicano del Carbono [PMC] (2015). Una REDD para SALVAR la SOMBRA de la Sierra Madre de Chiapas. Manual de Procedimientos Inventario de Biodiversidad. Recuperado de: http://pmcarbono.org/pmc/ descargas/proyectos/redd/MANUAL_Inventario_de_ Biodiversidad_1.0_Cafetales.pdf

Raunkiær, C. (1934). The Life Forms of Plants and Statistical Plant Geography. Oxford: Clarendon Press.

Rosenzweig, M. L. (1995). Species Diversity in Space and Time. Cambridge, UK: Cambridge University Press.

Rybicki, J., \& Hansi, I. (2013). Species-area relationships and extinctions caused by habitat loss and fragmentation. Ecology Letters, 16, 27-38. doi:10.1111/ele.12065

Saetersdal, M., \& I. Gjerde, (2011). Prioritising conservation areas using species surrogate measures: consistent with ecological theory? Journal of Applied Ecology, 48, 12361240. doi:10.1111/j.1365-2664.2011.02027.x

Sala, O. E., van Vuuren, D., Pereira, H. M., Lodge, D., Alder, J., Cumming, G., Dobson, A., Volters, W., Xenopoulos, M., \& Zaetsev, A. S. (2005). Biodiversity across scenarios. In S. Carpenter, L. P. Prabhu, E. M. Bennet \& M. B. Zurek (Eds.), Ecosystem and Human Well-Being Scenarios (pp. 375-408). Washington, DC: Island Press. 
Sánchez-González, A. y López-Mata, L. 2003. Clasificación y ordenación de la vegetación del norte de la Sierra Nevada, a lo largo de un gradiente altitudinal. Anales del Instituto de Biología, Universidad Nacional Autónoma de México, Serie Botánica, 74(1), 43-71.

Santi, E., Maccherini, S., Rocchini, D., Bonini, I., Brunialti, G., Favilli, L., Perini, C., Pezzo, F., Piazzini, S., Rota, E., Salerni, E., \& Chiarucci, A. (2010). Simple to sample: vascular plants as surrogate group in a nature reserve. Journal for Nature Conservation, 18(1), 2-11. doi:10.1016/j.jnc.2009.02.003

Scheiner, S. M. (2003). Six types of species-area curves. Global Ecology and Biogeography, 12, 441-447. doi:10.1046/j.1466-822X.2003.00061.x

Schoener, T. W. (1976). The species-area relationship within archipelagoes: models and evidence from island birds. (pp. 629-642). Proceedings of the XVI International Ornithological Congress 6.

Shugart, H. H. (1996). Plant and ecosystem functional types. In T. M. Smith, H. H. Shugart, \& F. I. Woodward (Eds.), Plant Functional Types: Their Relevance to Ecosystem Properties and Global Change (pp. 20-43). Cambridge, UK: Cambridge University Press.

Smith, A. B. (2010). Caution with curves: caveats for using the species-area relationship in conservation. Biological Conservation, 143(3), 555-564. doi:10.1016/j.biocon.2009.11.003

Smith, T. M., Shugart, H. H., Woodward, F. I. \& Burton, P. J. 1993. Plant functional types. In Solomon, A. M. \& Shugart, H. H. (Eds.), Vegetation dynamics and global change (pp. 272-292). New York, NY: Chapman and Hall.

Specht, A., \& Specht, R. L. (1993). Species richness and canopy productivity of Australian plant communities. Biodiversity and Conservation, 2, 152-167.

Specht, R. L. \& A. Specht. (2013). Australia, biodiversity of ecosystems. In S. A. Levin, (Ed.), Encyclopedia of Biodiversity (pp. 291-306). Amsterdam: Elsevier/Academic Press.
Specht, R. L. (1972). Water use by perennial evergreen plant communities in Australia and Papua New Guinea. Australian Journal of Botany, 20(3) 273-299. doi:10.1071/ BT9720273

Specht, R. L. (1994). Species richness of vascular plants and vertebrates in relation to canopy productivity. In: M. Arianoutsou, \& R. H. Groves, Plant-Animal Interactions in Mediterranean-Type Ecosystems (pp. 15-24). Netherlands: Kluwer Academic Publishers.

Specht, R. L. (2012). Biodiversity of Terrestrial Ecosystems in Tropical to Temperate Australia. International Journal of Ecology, 1-15. doi:10.1155/2012/359892

Specht, R. L., \& A. Specht. (1989). Species richness of sclerophyll (heathy) plant communities in Australia-the influence of overstorey cover. Australian Journal of Botany, 37, 337-350. doi:10.1071/bt9890337

Sprugel, D. G. (1983). Correcting for bias in log-transformed allometric equations. Ecology, 64(1), 209-210. doi:10.2307/1937343

Thompson, I., Mackey, B., McNulty, S., \& Mosseler, A. (2009). Forest Resilience, Biodiversity, and Climate Change. A synthesis of the biodiversity/resilience/stability relationship in forest ecosystems. Montreal, Canada: Secretariat of the Convention on Biological Diversity.

Tilman, D., \& Lehman, C. L. (1997). Habitat destruction and species extinctions. In D. Tilman, \& P. Kareiva (Eds.), Spatial Ecology: The Role of Space in Population Dynamics and Interspecific Interactions (pp. 233-249). New Jersey: Princeton University Press.

Tilman, D., Knops, J., Wedin, D., \& Reich, P. 2002. Plant diversity and composition: effects on productivity and nutrient dynamics of experimental grasslands. In: M. Loreau, S. Naeem, \& P. Inchausti (Eds.) Biodiversity and Ecosystem Functionals (pp. 21-35). Oxford, UK: Oxford University Press.

Tilman, D., May, R. M., Lehman, C. L., \& Nowak, M. A. (1994). Habitat destruction and the extinction debt. Nature, 371, 65-66. doi:10.1038/371065a0 
Tjørve, E. (2003). Shapes and functions of species-area curves: a review of possible models. Journal of Biogeography, 30, 827-835. doi:10.1046/j.1365-2699.2003.00877.x

Tjørve, E. (2009). Shapes and functions of species-area curves (II): a review of new models and parameterizations. Journal of Biogeography, 36, 1435-1445. doi: 10.1111/j.1365-2699.2009.02101.x

Tjørve, E. (2010). How to resolve the SLOSS debate: lessons from species-diversity models. Journal of Theoretical Biology, 264(2):604-12. doi: 10.1016/j.jtbi.2010.02.009

Triantis, K. A., Guilhaumon, F., \& Whittaker, R. J. (2012). The island species-area relationship: biology and statistics. Journal of Biogeography, 39, 215-231. doi:10.1111/j.1365-2699.2011.02652.x

Turner, W. R., \& Tjørve, E. (2005). Scale-dependence in species-area relationships. Ecography, 28(6), 721-730.

United Nations, Treaty Series [UNTC]. 1992. Convention on Biological Diversity. Rio de Janeiro: UNTC.

White, J. F., \& Gould, S. J. (1965). Interpretation of the coefficient in the allometric equation. The American Naturalist, 99(904), 5-18.

Williamson, M., Gaston, K. J., \& Lonsdale, W. M. (2001). The species-area relationship does not have an asymptote! Journal of Biogeography, 28, 827-830. doi:10.1046/j.1365-2699.2001.00603.x
Williamson, M., Gaston, K. J., \& Lonsdale, W. M. (2002). An asymptote is an asymptote and not found in species-area relationships. Journal of Biogeography, 29(12), 17131713. doi:10.1046/j.1365-2699.2002.00798.x

Wright, S. J. (1981). Intra-archipelago vertebrate distributions: the slope of the species-area relation. The American Naturalist, 118(5), 726-748.

Zhang, Z., Zhong, Q., Niklas, K. J., Cai, L., Yang, Y., \& Cheng, D. (2016). A predictive nondestructive model for the covariation of tree height, diameter and stem volume scaling relationships. Scientific Reports, 6(1), 1-9. doi:10.1038/srep31008

Manuscrito recibido el 14 de agosto de 2018

Aceptado el 09 de noviembre de 2020

Publicado el 29 de octubre de 2021

Este documento se debe citar como:

Sánchez-Sánchez, C., Paz-Pellat, F., Hernández-de la Rosa, P., Velázquez-Rodríguez, A., Vibrans, H., Vargas-Hernández, J., Valdez-Hernández, J. I., \& Valdez-Lazalde, J. R. (2021). Riqueza de especies y tipos funcionales: su relación en bosques de oyamel del Monte Tláloc, Estado de México. Madera y Bosques, 27(4), e2742427. doi: 10.21829/myb.2021.2742427

Madera y Bosques, por Instituto de Ecología, A.C. se distribuye bajo una Licencia Creative Commons Atribución-No Comercial-Compartir Igual 4.0 Internacional. 